\title{
The Randomized Iterate Revisited - Almost Linear Seed Length PRGs from A Broader Class of One-way Functions
}

\author{
$\mathrm{Yu} \mathrm{Yu*} \quad$ Dawu $\mathrm{Gu}^{\dagger} \quad$ Xiangxue $\mathrm{Li}^{\ddagger} \quad$ Jian Weng§
}

\begin{abstract}
We revisit "the randomized iterate" technique that was originally used by Goldreich, Krawczyk, and Luby (SICOMP 1993) and refined by Haitner, Harnik and Reingold (CRYPTO 2006) in constructing pseudorandom generators (PRGs) from regular one-way functions (OWFs). We abstract out a technical lemma (which is folklore in leakage resilient cryptography), and use it to provide a simpler and more modular proof for the Haitner-Harnik-Reingold PRGs from regular OWFs.

We introduce a more general class of OWFs called "weakly-regular one-way functions" from which we construct a PRG with seed length $O(n \cdot \log n)$. More specifically, consider an arbitrary one-way function $f$ with range divided into sets $\mathcal{Y}_{1}, \mathcal{Y}_{2}, \ldots, \mathcal{Y}_{n}$ where each $\mathcal{Y}_{i} \stackrel{\text { def }}{=}\left\{y: 2^{i-1} \leq\left|f^{-1}(y)\right|<2^{i}\right\}$. We say that $f$ is weakly-regular if there exists a (not necessarily efficient computable) cut-off point max such that $\mathcal{Y}_{\max }$ is of some noticeable portion (say $n^{-c}$ for constant $c$ ), and $\mathcal{Y}_{\max +1}, \ldots, \mathcal{Y}_{n}$ only sum to a negligible fraction. We construct a PRG by making $\tilde{O}\left(n^{2 c+1}\right)$ calls to $f$ and achieve seed length $O(n \cdot \log n)$ using bounded space generators. This generalizes the approach of Haitner et al., where regular OWFs fall into a special case for $c=0$. We use a proof technique that is similar to and extended from the method by Haitner, Harnik and Reingold for hardness amplification of regular weakly-one-way functions.

Our work further explores the feasibility and limits of the "randomized iterate" type of black-box constructions. In particular, the underlying $f$ can have an arbitrary structure as long as the set of images with maximal preimage size has a noticeable fraction. In addition, our construction is much more seed-length efficient and security-preserving (albeit less general) than the HILL-style generators where the best known construction by Vadhan and Zheng (STOC 2012) requires seed length $\tilde{O}\left(n^{3}\right)$.
\end{abstract}

Keywords: Foundations, Pseudorandom Generators, One-way Functions, the Randomized Iterate.

\footnotetext{
*Shanghai Jiaotong University. Email: yuyuathk@gmail.com.

${ }^{\dagger}$ Shanghai Jiaotong University.

${ }^{\ddagger}$ East China Normal University.

$\S$ Jinan University.
} 


\section{Introduction}

That one-way functions (OWFs) imply pseudorandom generators (PRGs) [13] is one of the central results upon which modern cryptography is successfully founded. The problem dates back to the early 80's when Blum, Micali [2] and Yao [19] independently observed that a PRG (often referred to as the BMY generator) can be efficiently constructed from one-way permutations (OWPs). That is, given a OWP $f$ on $n$-bit input $x$ and its hardcore predicate $h_{c}$ (e.g., by Goldreich and Levin [8]), a single invocation of $f$ already implies a PRG $g: x \mapsto\left(f(x), h_{c}(x)\right)$ with a stretch ${ }^{1}$ of $\Omega(\log n)$ bits and it extends to arbitrary stretch by repeated iterations (seen by a hybrid argument). Unfortunately, the BMY generator does not immediately apply to an arbitrary OWF since the output of $f$ might be of too small amount of entropy to be secure for subsequent iterations.

The Randomized iterate - PRGs from special OWFs. Goldreich, Krawczyk, and Luby [7] extended the BMY generator by inserting a randomized operation (using $k$-wise independent hash functions) into every two applications of $f$, from which they built a PRG of seed length $O\left(n^{3}\right)$ assuming that the underlying OWF is known-regular ${ }^{2}$. Haitner, Harnik and Reingold [11] further refined the approach (for which they coined the name "the randomized iterate") as in Figure 1 below, where in

$$
x_{1} \stackrel{f}{\longrightarrow} y_{1} \stackrel{h_{1}}{\longrightarrow} x_{2} \stackrel{f}{\longrightarrow} y_{2} \stackrel{h_{2}}{\longrightarrow} \cdots x_{k} \stackrel{f}{\longrightarrow} y_{k} \stackrel{h_{k}}{\longrightarrow}
$$

Figure 1: An illustration of the randomized iterate.

between every $i^{\text {th }}$ and $(i+1)^{\text {th }}$ iterations a random pairwise-independent hash function $h_{i}$ is applied. Haitner et al. [11] showed that, when $f$ is instantiated with any (possibly unknown) regular one-way function, it is hard to invert any $k^{\text {th }}$ iterate (i.e., recovering any $x_{k}$ s.t. $f\left(x_{k}\right)=y_{k}$ ) given $y_{k}$ and the description of the hash functions. This gives a PRG of seed length $O\left(n^{2}\right)$ by running the iterate $n+1$ times and outputting a hardcore bit at every iteration. The authors of [10] further derandomize the PRG by generating all the hash functions from bounded space generators (e.g., Nisan's generator [17]) using a seed of length $O(n \log n)$. Although the randomized iterate is mostly known for construction of PRGs from regular OWFs, the authors of [10] also introduced many other interesting applications such as linear seed length PRGs from any exponentially hard regular OWFs, $O\left(n^{2}\right)$ seed length PRGs from any exponentially hard OWFs, $O\left(n^{7}\right)$ seed length PRGs from any OWFs, and hardness amplification of regular weakly-OWFs. Dedic, Harnik and Reyzin [3] showed that the amount of secret randomness can be reduced to achieve tighter reductions, i.e., if a regular one-way function $f$ has $2^{k}$ images then the amount of secret randomness needed is $k$ (instead of $n$ bits). Yu et al. [20] further reduced the seed length of the PRG (based on any regular OWFs) to $O(\omega(1) \cdot n)$ for any efficiently computable $\omega(1)$.

The HILL Approach - PRGs from ANy OWFs. Håstad, Impagliazzo, Levin and Luby (HILL) [13] gave the seminal result that pseudorandom generators can be constructed from any one-way functions. Nevertheless, they only gave a complicated (and not practically efficient) construction of PRG with seed length $\tilde{O}\left(n^{10}\right)$ and sketched another one with seed length $\tilde{O}\left(n^{8}\right)$, which was formalized and proven in [14]. Haitner, Reingold, and Vadhan [12] introduced the notion of next-block pseudoentropy, and gave a construction of seed length $\tilde{O}\left(n^{4}\right)$. Vadhan and Zheng [18] further reduced the seed length of the uniform construction to $\tilde{O}\left(n^{3}\right)$, which is the current state-of-the-art.

A summaRY. The randomized iterate has advantages (over the HILL approach) such as shorter (almost linear) seed length and tighter reductions, but it remains open if the approach can be further generalized ${ }^{3}$

\footnotetext{
${ }^{1}$ The stretch of a PRG refers to the difference between output and input lengths (see Definition 2.3).

${ }^{2} \mathrm{~A}$ function $f(x)$ is regular if every image has the same number (say $\alpha$ ) of preimages, and it is known- (resp., unknown-) regular if $\alpha$ is efficiently computable (resp., inefficient to approximate) from the security parameter.

${ }^{3}$ The randomized iterate handles almost-regular one-way functions as well and this generalization is not hard to see
} 
(i.e., to go beyond regular one-way functions). In this paper, we answer this question by introducing a more general class of one-way functions and giving a construction based on the randomized iterate that enjoys seed length $O(n \cdot \log n)$ and tighter reductions.

A technical Lemma. First, we abstract out a technical lemma from [10] (see Lemma 3.1) that, informally speaking, "if any algorithm wins a one-sided game (e.g., inverting a OWF) on uniformly sampled challenges only with some negligible probability, then it cannot do much better (beyond a negligible advantage) in case that the challenges are sampled from any distribution of logarithmic Rényi entropy deficiency ${ }^{4}$. In fact, this lemma was implicitly known in leakage-resilient cryptography. Analogous observations were made in similar settings $[1,5,4]$, where either the game is two-sided (e.g., indistinguishability applications) or the randomness is sampled from slightly defected min-entropy source. Plugging this lemma into [10] immediately yields a simpler proof for the key lemma of [10] (see Lemma 3.2), namely, "any $k^{\text {th }}$ iterate (instantiated with a regular OWF) is hard-to-invert". The rationale is that $y_{k}$ has sufficiently high Rényi entropy (even conditioned on the hash functions) that is only logarithmically less than the ideal case where $y_{k}$ is uniform (over the range of $f$ ) and independent of the hash functions, which is hard to invert by the one-way-ness assumption.

The MAIN RESUlts. We introduce a class of one-way functions called weakly-regular one-way functions. Consider an arbitrary OWF $f:\{0,1\}^{n} \rightarrow\{0,1\}^{l}$ with range divided into sets $\mathcal{Y}_{1}, \ldots, \mathcal{Y}_{n}$, where each $\mathcal{Y}_{i} \stackrel{\text { def }}{=}\left\{y: 2^{i-1} \leq\left|f^{-1}(y)\right|<2^{i}\right\}$ and $\left|f^{-1}(y)\right|$ refers to preimage size of $y$ (i.e., the number of images that map to $y$ under $f$ ). We say that $f$ is weakly-regular if there exists an integer function $\max =\max (n)$ such that $\mathcal{Y}_{\max }$ is of some noticeable portion $\left(n^{-c}\right.$ for constant $\left.c\right)$, and $\mathcal{Y}_{\max +1}, \ldots, \mathcal{Y}_{n}$ only sum to a negligible fraction $\epsilon(n)$. Note that regular one-way functions fall into a special case for $c=0, \epsilon(n)=0$ and arbitrary (not necessarily efficient) function $\max (\cdot)$. We give a construction that only requires the knowledge about $c$ (i.e., oblivious of $\max$ and $\epsilon$ ). Informally speaking, as illustrated in Figure 1, the main idea is that for any round number $k$ conditioned on $y_{k} \in \mathcal{Y}_{\max }$ the Rényi entropy of $y_{k}$ given the hash functions is close to the ideal case where $f\left(U_{n}\right)$ hits $\mathcal{Y}_{\max }$ with noticeable probability (and is independent of the hash functions), which is hard to invert. We have by the pairwise independence (in fact, universality already suffices) of the hash functions that every $y_{k} \in \mathcal{Y}_{\max }$ is an independent event of probability $n^{-c}$. By a Chernoff bound, running the iterate $\Delta=n^{2 c} \cdot \omega(\log n)$ times yields that with overwhelming probability there is at least one occurrence of $y_{k} \in \mathcal{Y}_{\max }$, which implies every $\Delta$ iterations are hard-to-invert, i.e., for any $j=\operatorname{poly}(n)$ it is hard to predict $x_{1+(j-1) \Delta}$ given $y_{j \Delta}$ and the hash functions. A PRG follows by outputting $\log n$ hardcore bits for every $\Delta$ iterations and in total making $\tilde{O}\left(n^{2 c+1}\right)$ calls to $f$. This requires seed length $\tilde{O}\left(n^{2 c+2}\right)$, and can be pushed to $O(n \cdot \log n)$ bits using bounded space generators $[17,16]$, ideas borrowed from [10] with more significant reductions in seed length (we reduce by factor $\tilde{O}\left(n^{2 c+1}\right.$ ) whereas [10] saves factor $\left.\tilde{O}(n)\right)$. Overall, our technique is similar in spirit to the hardness amplification of regular weakly-one-way ${ }^{5}$ functions introduced by Haitner et al. in the same paper [10]. Roughly speaking, the idea was that for any inverting algorithm A, a weakly one-way function has a set that $A$ fails upon (the failing-set of $A$ ), and thus sufficiently many iterations are bound to hit every such failing-set to yield a strongly-one-way function (that is hard-to-invert on an overwhelming fraction). However, in our case the lack of a regular structure for the underlying function and the negligible fraction (i.e., $\mathcal{Y}_{\max +1}, \ldots, \mathcal{Y}_{n}$ ) further complicate the analysis (see Remark B.1 for some discussions), and we make our best effort to provide an intuitive and modular proof.

ON THE EFFICIENCY, FEASIBILITY AND LIMITS. From the application point of view, known-regular

(implicit in [10, 20]). Similarly, the construction we introduced in this paper only needs "weakly-almost-regular one-way functions" (of which almost-regular one-way functions fall into a special case). See Remark 2.1 for some discussions.

${ }^{4}$ The Rényi entropy deficiency of a random variable $W$ over set $\mathcal{W}$ refers to the difference between entropies of $U_{\mathcal{W}}$ and $W$, i.e., $\log |\mathcal{W}|-\mathbf{H}_{2}(W)$, where $U_{\mathcal{W}}$ denotes the uniform distribution over $\mathcal{W}$ and $\mathbf{H}_{2}(W)$ is the Rényi entropy of $W$.

${ }^{5}$ We should not confuse "weakly-regular" with "weakly-one-way", where the former "weakly" describes regularity (i.e., regular on a noticeable fraction as in Definition 2.4) and the latter is used for one-way-ness (i.e., hard-to-invert on a noticeable fraction [19]). 
one-way functions may already suffice for the following reasons:

1. If a one-way function behaves like a random function, then it is known(-almost)-regular. In other words, most functions are known(-almost)-regular. We state this as Lemma C.1 in Appendix C.

2. In practice, many one-way function candidates turn out to be known-regular or even 1-to-1. For example, Goldreich, Levin and Nisan [9] showed that 1-to-1 one-way functions can be based on concrete intractable problems such as RSA and DLP.

It is folklore (see, e.g., $[6,20])$ that pseudorandom generators can be constructed almost optimally from known(-almost)-regular one-way functions, i.e., with seed length $O(n \cdot \omega(1))$ and $O(\omega(1))$ nonadaptive OWF calls for any efficiently computable super-constant $\omega(1)$. Despite the aforementioned, the study on minimizing the knowledge required for the underlying one-way functions (and at the same time improving the efficiency of the resulting pseudorandom generator) is of theoretical significance, and it improves our understanding about feasibility and limits of black-box reductions. In particular, Holenstein and Sinha [15] showed that $\Omega(n / \log n)$ black-box calls to an arbitrary (including unknownregular) one-way function is necessary to construct a PRG, and Haitner, Harnik and Reingold [10] gave an explicit construction (from unknown-regular one-way functions) of seed length $O(n \cdot \log n)$ that matches this bound. In the most general setting, Håstad et al. [13] established the principle feasibility result that pseudorandom generators can based on any one-way functions but the current state-of-the-art [18] still requires seed length $\tilde{O}\left(n^{3}\right)$. We take a middle course by introducing weaklyregular one-way functions that lie in between regular one-way functions and arbitrary ones, and giving a construction of pseudorandom generator with seed length $O(n \cdot \log n)$ and using tighter reductions. We refer to Appendix $\mathrm{C}$ for a discussion about the gap between weakly one-way functions and arbitrary ones, namely, any one-way function that is not weakly-regular would be somewhat (arguably) artificial.

\section{Preliminaries}

\subsection{Notations and Definitions}

Notations. We use $[n]$ to denote set $\{1, \ldots, n\}$. We use capital letters (e.g., $X, Y$ ) for random variables, standard letters (e.g., $x, y$ ) for values, and calligraphic letters (e.g., $\mathcal{Y}, \mathcal{S}$ ) for sets. $|\mathcal{S}|$ denotes the cardinality of set $\mathcal{S}$. We use shorthand $\mathcal{Y}_{[n]} \stackrel{\text { def }}{=} \bigcup_{t=1}^{n} \mathcal{Y}_{t}$. For function $f:\{0,1\}^{n} \rightarrow\{0,1\}^{l(n)}$, we use shorthand $f\left(\{0,1\}^{n}\right) \stackrel{\text { def }}{=}\left\{f(x): x \in\{0,1\}^{n}\right\}$, and denote by $f^{-1}(y)$ the set of $y$ 's preimages under $f$, i.e. $f^{-1}(y) \stackrel{\text { def }}{=}\{x: f(x)=y\}$. We use $s \leftarrow S$ to denote sampling an element $s$ according to distribution $S$, and let $s \stackrel{\$}{\leftarrow}$ denote sampling $s$ uniformly from set $\mathcal{S}$, and let $y:=f(x)$ denote value assignment. We use $U_{n}$ and $U_{\mathcal{X}}$ to denote uniform distributions over $\{0,1\}^{n}$ and $\mathcal{X}$ respectively, and let $f\left(U_{n}\right)$ be the distribution induced by applying function $f$ to $U_{n}$. We use $\operatorname{CP}(X)$ to denote the collision probability of $X$, i.e., $\mathrm{CP}(X) \stackrel{\text { def }}{=} \sum_{x} \operatorname{Pr}[X=x]^{2}$, and denote by $\mathbf{H}_{2}(X) \stackrel{\text { def }}{=}-\log \mathrm{CP}(X)$ the Rényi entropy. We also define conditional Rényi entropy (and probability) of a random variable $X$ conditioned on another random variable $Z$ by

$$
\mathbf{H}_{2}(X \mid Z) \stackrel{\text { def }}{=}-\log (\mathrm{CP}(X \mid Z)) \stackrel{\text { def }}{=}-\log \left(\mathbb{E}_{z \leftarrow Z}\left[\sum_{x} \operatorname{Pr}[X=x \mid Z=z]^{2}\right]\right)
$$

A function negl $: \mathbb{N} \rightarrow[0,1]$ is negligible if for every constant $c$ we have negl $(n)<n^{-c}$ holds for all sufficiently large $n$ 's, and a function $\mu: \mathbb{N} \rightarrow[0,1]$ is called noticeable if there exists constant $c$ such that $\mu(n) \geq n^{-c}$ for all sufficiently large $n$ 's.

We define the computational distance between distribution ensembles $X \stackrel{\text { def }}{=}\left\{X_{n}\right\}_{n \in \mathbb{N}}$ and $Y \stackrel{\text { def }}{=}$ $\left\{Y_{n}\right\}_{n \in \mathbb{N}}$, denoted by $\mathrm{CD}_{T(n)}(X, Y) \leq \varepsilon(n)$, if for every probabilistic distinguisher $\mathrm{D}$ of running time 
$T(n)$ it holds that

$$
\left|\operatorname{Pr}\left[\mathrm{D}\left(1^{n}, X_{n}\right)=1\right]-\operatorname{Pr}\left[\mathrm{D}\left(1^{n}, Y_{n}\right)=1\right]\right| \leq \varepsilon(n) .
$$

The statistical distance between $X$ and $Y$, denoted by $\operatorname{SD}(X, Y)$, is defined by

$$
\mathrm{SD}(X, Y) \stackrel{\text { def }}{=} \frac{1}{2} \sum_{x}|\operatorname{Pr}[X=x]-\operatorname{Pr}[Y=x]|=\mathrm{CD}_{\infty}(X, Y)
$$

We use $\mathrm{SD}(X, Y \mid Z)\left(\operatorname{resp} . \mathrm{CD}_{t}(X, Y \mid Z)\right)$ as shorthand for $\mathrm{SD}((X, Z),(Y, Z))\left(\operatorname{resp} . \mathrm{CD}_{t}((X, Z),(Y, Z))\right)$. Simplifying Assumptions and Notations. To simplify the presentation, we make the following assumptions without loss of generality. It is folklore that one-way functions can be assumed to be length-preserving (see [11] for full proofs). Throughout, most parameters are functions of the security parameter $n$ (e.g., $T(n), \varepsilon(n), \alpha(n))$ and we often omit $n$ when clear from the context (e.g., $T, \varepsilon, \alpha$ ). By notation $f:\{0,1\}^{n} \rightarrow\{0,1\}^{l}$ we refer to the ensemble of functions $\left\{f_{n}:\{0,1\}^{n} \rightarrow\{0,1\}^{l(n)}\right\}_{n \in \mathbb{N}}$. As slight abuse of notion, poly might be referring to the set of all polynomials or a certain polynomial, and $h$ might be either a function or its description, which will be clear from the context.

Definition 2.1 (pairwise independent hashing) A family of hash functions $\mathcal{H} \stackrel{\text { def }}{=}\left\{h:\{0,1\}^{n} \rightarrow\right.$ $\left.\{0,1\}^{m}\right\}$ is pairwise independent if for any $x_{1} \neq x_{2} \in\{0,1\}^{n}$ and any $v \in\{0,1\}^{2 m}$ it holds that $\operatorname{Pr}_{h \leftarrow \mathcal{H}}\left[\left(h\left(x_{1}\right), h\left(x_{2}\right)\right)=v\right]=2^{-2 m}$, or equivalently, $\left(H\left(x_{1}\right), H\left(x_{2}\right)\right)$ is i.i.d. to $U_{2 m}$ where $H$ is uniform over $\mathcal{H}$. It is well known that there are efficiently computable families of pairwise independent hash functions of description length $\Theta(n+m)$.

Definition 2.2 (one-way functions) A function $f:\{0,1\}^{n} \rightarrow\{0,1\}^{l(n)}$ is $(T(n), \varepsilon(n)$ )-one-way if $f$ is polynomial-time computable and for any probabilistic algorithm $\mathrm{A}$ of running time $T(n)$

$$
\operatorname{Pr}_{y \leftarrow f\left(U_{n}\right)}\left[\mathrm{A}\left(1^{n}, y\right) \in f^{-1}(y)\right] \leq \varepsilon(n) .
$$

We say that $f$ is a (strongly) one-way function if $T(n)$ and $1 / \varepsilon(n)$ are both super-polynomial in $n$.

Definition 2.3 (pseudorandom generators [2, 19]) A deterministic function $g:\{0,1\}^{n} \rightarrow\{0,1\}^{n+s(n)}$ $(s(n)>0)$ is a $(T(n), \varepsilon(n))$-secure PRG with stretch $s(n)$ if $g$ is polynomial-time computable and

$$
\mathrm{CD}_{T(n)}\left(g\left(1^{n}, U_{n}\right), U_{n+s(n)}\right) \leq \varepsilon(n) .
$$

We say that $g$ is a pseudorandom generator if $T(n)$ and $1 / \varepsilon(n)$ are both super-polynomial in $n$.

Definition 2.4 (weakly-regular one-way functions) Let $f:\{0,1\}^{n} \rightarrow\{0,1\}^{l(n)}$ be a one-way function. For every $n \in \mathbb{N}$ divide range $f\left(\{0,1\}^{n}\right)$ into sets $\mathcal{Y}_{1}, \ldots, \mathcal{Y}_{n}$ (i.e., $\mathcal{Y}_{1} \cup \ldots \cup \mathcal{Y}_{n}=f\left(\{0,1\}^{n}\right)$ ) where $\mathcal{Y}_{j} \stackrel{\text { def }}{=}\left\{y: 2^{j-1} \leq\left|f^{-1}(y)\right|<2^{j}\right\}$ for every $1 \leq j \leq n$. We say that $f$ is weakly-regular if there exist constant $c$, integer function $\max =\max (n)$, and negligible function $\epsilon=\epsilon(n)$ such that the following holds for all sufficiently large n's :

$$
\begin{gathered}
\operatorname{Pr}\left[f\left(U_{n}\right) \in \mathcal{Y}_{\max }\right] \geq n^{-c}, \\
\operatorname{Pr}\left[f\left(U_{n}\right) \in\left(\mathcal{Y}_{\max +1} \cup \mathcal{Y}_{\max +2} \cup \ldots \cup \mathcal{Y}_{n}\right)\right] \leq \epsilon,
\end{gathered}
$$

Note that $\max (\cdot)$ can be arbitrary (not necessarily efficient) functions and thus regular one-way functions fall into a special case for $c=0$. 
Remark 2.1 (on further categorization and generalization.) We can further divide the above class of functions into weakly-known-regular and weakly-unknown-regular one-way functions depending on whether $\max (\cdot)$ is efficiently computable or not. This is however not necessary since our construction needs no knowledge about $\max (\cdot)$ and thus handles any weakly-regular one-way functions. In fact, our construction only assumes that $f$ is weakly-almost-regular, i.e., for some $d=d(n) \in O(\log n)$ it holds that

$$
\operatorname{Pr}\left[f\left(U_{n}\right) \in\left(\mathcal{Y}_{\max -d} \cup \mathcal{Y}_{\max -d+1} \cup \ldots \cup \mathcal{Y}_{\max }\right)\right] \geq n^{-c}
$$

instead of (1), where almost-regular one-way functions become a special case for $c=0$. We mainly give the proof under the assumption of Definition 2.4 for neatness, and sketch how to adapt the proof to the weakly-almost-regular case in Remark B.2 (see Appendix B).

\subsection{Technical Tools}

Theorem 2.1 (Goldreich-Levin Theorem [8]) Let $(X, Y)$ be a distribution ensemble over $\left\{\{0,1\}^{n} \times\right.$ $\left.\{0,1\}^{\text {poly }(n)}\right\}_{n \in \mathbb{N}}$. Assume that for any PPT algorithm A of running time $T(n)$ it holds that

$$
\operatorname{Pr}\left[\mathrm{A}\left(1^{n}, Y\right)=X\right] \leq \varepsilon(n)
$$

Then, for any efficiently computable $m=m(n) \leq n$, there exists an efficient function family $\mathcal{H}_{c} \stackrel{\text { def }}{=}$ $\left\{h_{c}:\{0,1\}^{n} \rightarrow\{0,1\}^{m}\right\}$ of description size $\Theta(n)^{6}$, such that

$$
\mathrm{CD}_{T^{\prime}(n)}\left(H_{c}(X), U_{m} \mid Y, H_{c}\right) \in O\left(2^{m} \cdot(n \cdot \varepsilon)^{\frac{1}{3}}\right) .
$$

where $T^{\prime}(n)=T(n) \cdot(\varepsilon(n) / n)^{O(1)}$, and $H_{c}$ is the uniform distribution over $\mathcal{H}_{c}$.

Definition 2.5 (bounded-width layered branching program - LBP) An $(s, k, v)-L B P M$ is a finite directed acyclic graph whose nodes are partitioned into $k+1$ layers indexed by $\{1, \ldots, k+1\}$. The first layer has a single node (the source), the last layer has two nodes (sinks) labeled with 0 and 1 , and each of the intermediate layers has up to $2^{s}$ nodes. Each node in the $i \in[k]$ layer has exactly $2^{v}$ outgoing labeled edges to the $(i+1)^{\text {th }}$ layer, one for every possible string $h_{i} \in\{0,1\}^{v}$.

An equivalent (and perhaps more intuitive) model to the above is bounded space computation. That is, we assign labels to graph nodes (instead of associating them with the edges), at each $i^{\text {th }}$ layer the program performs arbitrary computation on the current node (labelled by $s$-bit string) and the current $v$-bit input $h_{i}$, advances (and assigns value) to a node in the $(i+1)^{t h}$ layer, and repeats until it reaches the last layer to produce the final output bit.

Theorem 2.2 (bounded-space generator [17, 16]) Let $s=s(n), k=k(n), v=v(n) \in \mathbb{N}$ and $\varepsilon=$ $\varepsilon(n) \in(0,1)$ be polynomial-time computable functions. Then, there exist a polynomial-time computable function $q=q(n) \in \Theta(v+(s+\log (k / \varepsilon)) \log k)$ and a generator $B S G:\{0,1\}^{q} \rightarrow\{0,1\}^{k \cdot v}$ that runs in time poly $(s, k, v, \log (1 / \varepsilon))$, and $\varepsilon$-fools every $(s, k, v)$ - LBP $M$, i.e.,

$$
\left|\operatorname{Pr}\left[M\left(U_{k \cdot v}\right)=1\right]-\operatorname{Pr}\left[M\left(B S G\left(U_{n}\right)\right)=1\right]\right| \leq \varepsilon .
$$

\footnotetext{
${ }^{6}$ For example (see [8]), we can use an $m \times n$ Toeplitz matrix $a_{m, n}$ to describe the function family, i.e., $\mathcal{H}_{c} \stackrel{\text { def }}{=}$ $\left\{h_{c}(x) \stackrel{\text { def }}{=} a_{m, n} \cdot x, x \in\{0,1\}^{n}, a_{m, n} \in\{0,1\}^{m+n-1}\right\}$.
} 


\section{Pseudorandom Generators from Regular One-way Functions}

\subsection{A Technical Lemma}

Before we revisit the randomized iterate based on regular one-way functions, we introduce a technical lemma that simplifies the analysis in [10] and is also used to prove our main theorem in Section 4. Informally, it states that if any one-sided game (one-way functions, MACs, and digital signatures) is $(T, \varepsilon)$-secure on uniform secret randomness, then it will be $\left(T, \sqrt{2^{e+2} \cdot \varepsilon}\right)$-secure when the randomness is sampled from any distribution with $e$ bits of Rényi entropy deficiency.

Lemma 3.1 (one-sided game on imperfect randomness) For any $e \leq m \in \mathbb{N}$, let $\mathcal{W} \times \mathcal{Z}$ be any set with $|\mathcal{W}|=2^{m}$, let $\mathrm{Adv}: \mathcal{W} \times \mathcal{Z} \rightarrow[0,1]$ be any (deterministic) real-valued function, let $(W, Z)$ be any joint random variables over set $\mathcal{W} \times \mathcal{Z}$ satisfying $\mathbf{H}_{2}(W \mid Z) \geq m-e$, we have

$$
\mathbb{E}[\operatorname{Adv}(W, Z)] \leq \sqrt{2^{e+2} \cdot \mathbb{E}\left[\operatorname{Adv}\left(U_{\mathcal{W}}, Z\right)\right]}
$$

where $U_{\mathcal{W}}$ denotes uniform distribution over $\mathcal{W}$ (independent of $Z$ and any other distributions).

Proof. For any given $\delta$ define $\mathcal{S}_{\delta} \stackrel{\text { def }}{=}\left\{(w, z): \operatorname{Pr}[W=w \mid Z=z] \geq 2^{-(m-e)} / \delta\right\}$

$$
\begin{aligned}
& 2^{-(m-e)} \geq \sum_{z} \operatorname{Pr}[Z=z] \sum_{w} \operatorname{Pr}[W=w \mid Z=z]^{2} \\
& \geq \sum_{z} \operatorname{Pr}[Z=z] \sum_{w:(w, z) \in \mathcal{S}_{\delta}} \operatorname{Pr}[W=w \mid Z=z] \cdot 2^{-(m-e)} / \delta \\
& \geq \quad\left(2^{-(m-e)} / \delta\right) \cdot \operatorname{Pr}\left[(W, Z) \in \mathcal{S}_{\delta}\right],
\end{aligned}
$$

and thus $\operatorname{Pr}\left[(W, Z) \in \mathcal{S}_{\delta}\right] \leq \delta$. It follows that

$$
\begin{aligned}
\mathbb{E}[\operatorname{Adv}(W, Z)] & =\sum_{(w, z) \in \mathcal{S}_{\delta}} \operatorname{Pr}[(W, Z)=(w, z)] \cdot \operatorname{Adv}(w, z)+\sum_{(w, z) \notin \mathcal{S}_{\delta}} \operatorname{Pr}[Z=z] \cdot \operatorname{Pr}[W=w \mid Z=z] \cdot \operatorname{Adv}(w, z) \\
& \leq \sum_{(w, z) \in \mathcal{S}_{\delta}} \operatorname{Pr}[(W, Z)=(w, z)]+\left(2^{e} / \delta\right) \cdot \sum_{(w, z) \notin \mathcal{S}_{\delta}} \operatorname{Pr}[Z=z] \cdot 2^{-m} \cdot \operatorname{Adv}(w, z) \\
& \leq \delta+\left(2^{e} / \delta\right) \cdot \mathbb{E}\left[\operatorname{Adv}\left(U_{\mathcal{W}}, Z\right)\right],
\end{aligned}
$$

and we complete the proof by setting $\delta=\sqrt{2^{e} \cdot \mathbb{E}\left[\operatorname{Adv}\left(U_{\mathcal{W}}, Z\right)\right]}$.

On how to use the lemma. One can think of $\operatorname{Adv}(w, z)$ as the advantage of any specific adversary conditioned on the challenge being $w$ and the additional side information being $z$ (e.g., hash functions that are correlated to the challenges). Thus, the left-hand of (3) gives the adversary's advantage on slightly defected random source in consideration, which is bounded by the ideal case on the right-hand of (3), namely, the advantage on uniformly sampled challenges, such as a uniform random $y \leftarrow f\left(U_{n}\right)$ (for some regular one-way function $f$ ) independent of the hash functions.

\subsection{The Randomized Iterate}

Definition 3.1 (the randomized iterate $[10,7]$ ) Let $n \in \mathbb{N}$, let $f:\{0,1\}^{n} \rightarrow\{0,1\}^{n}$ be a lengthpreserving function, and let $\mathcal{H}$ be a family of pairwise-independent length-preserving hash functions over $\{0,1\}^{n}$. For $k \in \mathbb{N}, x_{1} \in\{0,1\}^{n}$ and vector $\vec{h}^{k}=\left(h_{1}, \ldots, h_{k}\right) \in \mathcal{H}^{k}$, recursively define the $k^{\text {th }}$ randomized iterate by:

$$
y_{k}=f\left(x_{k}\right), x_{k+1}=h_{k}\left(y_{k}\right)
$$


For $k-1 \leq t \in \mathbb{N}$, we denote the $k^{\text {th }}$ iterate by function $f^{k}$, i.e., $y_{k}=f^{k}\left(x_{1}, \vec{h}^{t}\right)$, where $\vec{h}^{t}$ is possibly redundant as $y_{k}$ only depends on $\vec{h}^{k-1}$.

The randomized version refers to the case where $x_{1} \stackrel{\$}{\leftarrow}\{0,1\}^{n}$ and $\vec{h}^{k-1} \stackrel{\$}{\leftarrow} \mathcal{H}^{k-1}$.

The derandomized version refers to that $x_{1} \stackrel{\$}{\leftarrow}\{0,1\}^{n}, \vec{h}^{k-1} \leftarrow B S G\left(U_{q}\right)$, where $q \in O(n \cdot \log n)$, $B S G:\{0,1\}^{q} \rightarrow\{0,1\}^{(k-1) \cdot \log |\mathcal{H}|}$ is a bounded-space generator ${ }^{7}$ that $2^{-2 n}$-fools every $(2 n+1, k, \log |\mathcal{H}|)$ $L B P$, and $\log |\mathcal{H}|$ is the description length of $\mathcal{H}$ (e.g., $2 n$ bits for concreteness).

Theorem 3.1 (PRGs from Regular OWFs [10]) For $n \in \mathbb{N}, k \in[n+1]$, let $f, \mathcal{H}$, $f^{k}$ and $B S G(\cdot)$ be as defined in Definition 3.1, and let $\mathcal{H}_{c}=\left\{h_{c}:\{0,1\}^{n} \rightarrow\{0,1\}\right\}$ be a family of Goldreich-Levin predicates, where $\mathcal{H}$ and $\mathcal{H}_{c}$ both have description length $\Theta(n)$. We define $G:\{0,1\}^{n} \times \mathcal{H}^{n} \times \mathcal{H}_{c} \rightarrow$ $\{0,1\}^{n+1} \times \mathcal{H}^{n} \times \mathcal{H}_{c}$ and $G^{\prime}:\{0,1\}^{n} \times\{0,1\}^{q(n)} \times \mathcal{H}_{c} \rightarrow\{0,1\}^{n+1} \times\{0,1\}^{q(n)} \times \mathcal{H}_{c}$ as below:

$$
\begin{gathered}
G\left(x_{1}, \vec{h}^{n}, h_{c}\right)=\left(h_{c}\left(x_{1}\right), h_{c}\left(x_{2}\right), \ldots, h_{c}\left(x_{n+1}\right), \vec{h}^{n}, h_{c}\right) . \\
G^{\prime}\left(x_{1}, u, h_{c}\right)=G\left(x_{1}, B S G(u), h_{c}\right) .
\end{gathered}
$$

Assume that $f$ is a regular (length-preserving) one-way function and that $B S G(\cdot), \mathcal{H}$ and $\mathcal{H}_{c}$ are efficient. Then, $G$ and $G^{\prime}$ are pseudorandom generators.

Proof Sketch of Theorem 3.1. It suffices to prove Lemma 3.2. Namely, for any $1 \leq k \leq n+1$, given $y_{k}$ and the hash functions (either sampled uniformly or from bounded space generators), it is hard to recover any $x_{k}$ satisfying $f\left(x_{k}\right)=y_{k}$. Then, Goldreich-Levin Theorem yields that each $h_{c}\left(x_{k}\right)$ is computationally unpredictable given $y_{k}$, which (together with $\vec{h}^{n}$ ) efficiently determines all the subsequent $h_{c}\left(x_{k+1}\right), \ldots, h_{c}\left(x_{n+1}\right)$. We complete the proof by Yao's "next/previous bit unpredictability implies pseudorandomness" argument [19]. It thus remains to prove Lemma 3.2 below which summarizes the statements of Lemma 3.2, Lemma 3.4, Lemma 3.11 from [11], and we provide a simpler proof.

Lemma 3.2 (the $k^{\text {th }}$ iterate is hard-to-invert) For any $n \in \mathbb{N}, k \in[n+1]$, let $f$, $\mathcal{H}$, $f^{k}$ be as defined in Definition 3.1. Assume that $f$ is a $(T, \varepsilon)$ regular one-way function, i.e., for every PPT A and $\mathrm{A}^{\prime}$ of running time $T$ it holds that

$$
\begin{aligned}
& \operatorname{Pr}\left[\mathrm{A}\left(f\left(U_{n}\right), \vec{H}^{n}\right) \in f^{-1}\left(f\left(U_{n}\right)\right)\right] \leq \varepsilon . \\
& \operatorname{Pr}\left[\mathrm{A}^{\prime}\left(f\left(U_{n}\right), U_{q}\right) \in f^{-1}\left(f\left(U_{n}\right)\right)\right] \leq \varepsilon .
\end{aligned}
$$

Then, for every such $\mathrm{A}$ and $\mathrm{A}^{\prime}$ it holds that

$$
\begin{gathered}
\operatorname{Pr}\left[\mathrm{A}\left(Y_{k}, \vec{H}^{n}\right) \in f^{-1}\left(Y_{k}\right)\right] \leq 2 \sqrt{k \cdot \varepsilon}, \\
\operatorname{Pr}\left[\mathrm{A}^{\prime}\left(Y_{k}^{\prime}, U_{q}\right) \in f^{-1}\left(Y_{k}^{\prime}\right)\right] \leq 2 \sqrt{(k+1) \cdot \varepsilon},
\end{gathered}
$$

where $Y_{k}=f^{k}\left(X_{1}, \vec{H}^{n}\right), Y_{k}^{\prime}=f^{k}\left(X_{1}, B S G\left(U_{q}\right)\right), X_{1}$ is uniform over $\{0,1\}^{n}$ and $\vec{H}^{n}$ is uniform over $\mathcal{H}^{n}$.

A simpler proof of Lemma 3.2 via Lemma 3.1. To apply Lemma 3.1, let $\mathcal{W}=f\left(\{0,1\}^{n}\right)$, $\mathcal{Z}=\mathcal{H}^{n}$, let $(W, Z)=\left(Y_{k}, \vec{H}^{n}\right), U_{\mathcal{W}}=f\left(U_{n}\right)$, and define

$$
\operatorname{Adv}\left(y, \vec{h}^{n}\right) \stackrel{\text { def }}{=}\left\{\begin{array}{l}
1, \text { if } \mathrm{A}\left(y, \vec{h}^{n}\right) \in f^{-1}(y) \\
0, \text { if } \mathrm{A}\left(y, \vec{h}^{n}\right) \notin f^{-1}(y)
\end{array}\right.
$$

\footnotetext{
${ }^{7}$ Such efficient generators exist by Theorem 2.2 , setting $s(n)=2 n+1, k(n)=\operatorname{poly}(n), v(n)=\log |\mathcal{H}|$ and $\varepsilon(n)=2^{-2 n}$ and thus $q(n)=O(n \cdot \log n)$.
} 
where $\mathrm{A}$ is assumed to be deterministic without loss of generality ${ }^{8}$. We have by Lemma 3.3 that

$$
\mathbf{H}_{2}\left(Y_{k} \mid \vec{H}^{n}\right) \geq \mathbf{H}_{2}\left(f\left(U_{n}\right) \mid \vec{H}^{n}\right)-\log k
$$

and thus Lemma 3.1 yields that

$$
\left.\operatorname{Pr}\left[\mathrm{A}\left(Y_{k}, \vec{H}^{n}\right) \in f^{-1}\left(Y_{k}\right)\right] \leq 2 \sqrt{k \cdot \operatorname{Pr}\left[\mathrm{A}\left(f\left(U_{n}\right), \vec{H}^{n}\right) \in f^{-1}\left(f\left(U_{n}\right)\right)\right.}\right] \leq 2 \sqrt{k \cdot \varepsilon} .
$$

The proof for (5) is similar except for setting ( $\left.W=Y_{k}^{\prime}, Z=U_{q}\right)$ and $\operatorname{letting} \operatorname{Adv}(y, u)=1$ iff $\mathrm{A}^{\prime}(y, u) \in f^{-1}(y)$. We have by Lemma 3.3 that

$$
\mathbf{H}_{2}\left(Y_{k}^{\prime} \mid U_{q}\right) \geq \mathbf{H}_{2}\left(f\left(U_{n}\right) \mid U_{q}\right)-\log (k+1)
$$

and thus we apply Lemma 3.1 to get

$$
\operatorname{Pr}\left[\mathrm{A}^{\prime}\left(Y_{k}^{\prime}, U_{q}\right) \in f^{-1}\left(Y_{k}^{\prime}\right)\right] \leq 2 \sqrt{(k+1) \cdot \operatorname{Pr}\left[\mathrm{A}^{\prime}\left(f\left(U_{n}\right), U_{q}\right) \in f^{-1}\left(f\left(U_{n}\right)\right)\right]} \leq 2 \sqrt{(k+1) \cdot \varepsilon} .
$$

The proof of Lemma 3.3 below appeared in [10], and we include it in Appendix A for completeness.

Lemma 3.3 (Rényi entropy conditions [10]) For the same assumptions as in Lemma 3.2, it holds that

$$
\begin{gathered}
\mathrm{CP}\left(f\left(U_{n}\right)\right)=\mathrm{CP}\left(f\left(U_{n}\right) \mid \vec{H}^{n}\right)=\mathrm{CP}\left(f\left(U_{n}\right) \mid B S G\left(U_{q}\right), U_{q}\right)=\frac{1}{\left|f\left(\{0,1\}^{n}\right)\right|}, \\
\mathrm{CP}\left(Y_{k} \mid \vec{H}^{n}\right) \leq \frac{k}{\left|f\left(\{0,1\}^{n}\right)\right|}, \\
\operatorname{CP}\left(Y_{k}^{\prime} \mid U_{q}\right) \leq \frac{k+1}{\left|f\left(\{0,1\}^{n}\right)\right|} .
\end{gathered}
$$

\section{A More General Construction of Pseudorandom Generators}

In this section we construct a pseudorandom generator with seed length $O(n \log n)$ from weakly-regular one-way functions (see Definition 2.4). We first show how to construct the PRG by running the iterate $\tilde{O}\left(n^{2 c+1}\right)$ times, and thus require large amount of randomness (i.e., $\tilde{O}\left(n^{2 c+2}\right)$ bits) to sample the hash functions. Then, we show the derandomized version where the amount of the randomness is compressed into $O(n \log n)$ bits using bounded space generators.

\subsection{The Randomized Version: A PRG with Seed Length $\tilde{O}\left(n^{2 c+2}\right)$}

Recall that any one-way function $f$ can be assumed to be length-preserving without loss of generality.

Theorem 4.1 (the randomized version) For $n, k \in \mathbb{N}$, assume that $f$ is a weakly-regular one-way function (with $c$, max and $\epsilon$ as defined in Definition 2.4), let $\mathcal{H}$ and $f^{k}$ be defined as in Definition 3.1, and let $\mathcal{H}_{c}=\left\{h_{c}:\{0,1\}^{n} \rightarrow\{0,1\}^{2 \log n}\right\}$ be a family of Goldreich-Levin hardcore functions. Then, for any efficient $\alpha=\alpha(n) \in \omega(1), \Delta=\Delta(n)=\alpha \cdot \log n \cdot n^{2 c}$ and $r=r(n)=\lceil n / \log n\rceil$, the function $g:\{0,1\}^{n} \times \mathcal{H}^{r \Delta-1} \times \mathcal{H}_{c} \rightarrow\{0,1\}^{2 n} \times \mathcal{H}^{r \Delta-1} \times \mathcal{H}_{c}$ defined as

$$
g\left(x_{1}, \vec{h}^{r \cdot \Delta-1}, h_{c}\right)=\left(h_{c}\left(x_{1}\right), h_{c}\left(x_{1+\Delta}\right), h_{c}\left(x_{1+2 \Delta}\right), \ldots, h_{c}\left(x_{1+r \cdot \Delta}\right), \vec{h}^{r \cdot \Delta-1}, h_{c}\right)
$$

is a pseudorandom generator.

Notice that a desirable property is that a construction assuming a sufficiently large $c$ works with any one-way function whose actual parameter is less than or equal to $c$.

\footnotetext{
${ }^{8}$ If $\mathrm{A}$ is probabilistic, let $\operatorname{Adv}\left(y, \vec{h}^{n}\right)=\operatorname{Pr}\left[\mathrm{A}\left(y, \vec{h}^{n}\right) \in f^{-1}(y)\right]$, where probability is taken over the internal coins of $\mathrm{A}$.
} 
Proof. The proof is similar to Theorem 3.1 based on Yao's hybrid argument [19]. Namely, the pseudorandomness of a sequence (with polynomially many blocks) is equivalent to that every block is pseudorandom conditioned on its suffix (or prefix). By the Goldreich-Levin Theorem and Lemma 4.1 below we know that every $h_{c}\left(x_{1+j \Delta}\right)$ is pseudorandom conditioned on $h_{c}, y_{(j+1) \Delta}$ and $\vec{h}^{r \Delta-1}$, which efficiently implies all subsequent blocks $h_{c}\left(x_{1+(j+1) \Delta}\right), \ldots, h_{c}\left(x_{1+r \Delta}\right)$. This completes the proof.

Lemma 4.1 (every $\Delta$ iterations are hard-to-invert) For $n, k \in \mathbb{N}$, let $f$ be a weakly-regular $(T, \varepsilon)$ OWF (with $c$ as defined in Definition 2.4), and let $\mathcal{H}, f^{k}, \alpha=\alpha(n), \Delta=\Delta(n)$ and $r=r(n)$ be as defined in Theorem 4.1. Then, for every $j \in[r]$, and for every PPT A of running time $T(n)-n^{O(1)}$ (for some universal constant $O(1)$ ) it holds that

$$
\operatorname{Pr}_{x_{1} \stackrel{\$}{\leftarrow}\{0,1\}^{n}, \vec{h}^{r \Delta-1} \stackrel{\$}{\leftarrow} \mathcal{H}^{r \Delta-1}}\left[\mathrm{~A}\left(y_{j \cdot \Delta}, \vec{h}^{r \Delta-1}\right)=x_{1+(j-1) \Delta}\right] \in O\left(n^{3 c / 2} \cdot r \cdot \Delta^{2} \cdot \sqrt{\varepsilon}\right) .
$$

Proof Sketch of Lemma 4.1. Assume towards a contradiction that

$$
\exists j^{*} \in[r], \exists \operatorname{PPT~A~}: \operatorname{Pr}\left[\mathrm{A}\left(Y_{j^{*} \cdot \Delta}, \vec{H}^{r \Delta-1}\right)=X_{1+\left(j^{*}-1\right) \Delta}\right] \geq \varepsilon_{\mathrm{A}}
$$

for some non-negligible function $\varepsilon_{\mathrm{A}}=\varepsilon_{\mathrm{A}}(n)$. Then, we build an efficient algorithm $\mathrm{M}^{\mathrm{A}}$ (see Algorithm 1) that invokes $\mathrm{A}$ and inverts $f$ with probablity $\Omega\left(\varepsilon_{\mathrm{A}}^{2} / n^{3 c} \cdot r^{2} \cdot \Delta^{4}\right.$ ) (as shown in Lemma 4.3), which is a contradiction to the $(T, \varepsilon)$-one-wayness of $f$ and thus completes the proof.

We define the events $\mathcal{E}_{k}$ and $\mathcal{S}_{k}$ in Definition 4.1 below, where $\mathcal{S}_{k}$ refers to that during the first $k$ iterates no $y_{t}(1 \leq t \leq k)$ hits the negligible fraction region (see Remark B.1 in Appendix B for the underlying intuitions), and $\mathcal{E}_{k}$ defines the desirable event that $y_{k}$ hits $\mathcal{Y}_{\max }$ (which implies the hard-toinvertness).

Definition 4.1 (events $\mathcal{S}_{k}$ and $\mathcal{E}_{k}$ ) For any $n \in \mathbb{N}$ and any $k \leq r \Delta$, define events

$$
\begin{gathered}
\mathcal{S}_{k} \stackrel{\text { def }}{=}\left(\left(X_{1}, \vec{H}^{r \Delta-1}\right) \in\left\{\left(x_{1}, \vec{h}^{r \Delta-1}\right): \forall t \in[k] \text { satisfies } y_{t} \in \mathcal{Y}_{[\max ]} \text {, where } y_{t}=f^{t}\left(x_{1}, \vec{h}^{r \Delta-1}\right)\right\}\right) \\
\mathcal{E}_{k} \stackrel{\text { def }}{=}\left(\left(X_{1}, \vec{H}^{r \Delta-1}\right) \in\left\{\left(x_{1}, \vec{h}^{r \Delta-1}\right): y_{k} \in \mathcal{Y}_{\max }, \text { where } y_{k}=f^{k}\left(x_{1}, \vec{h}^{r \Delta-1}\right)\right\}\right)
\end{gathered}
$$

where $\mathcal{Y}_{[\max ]}=\mathcal{Y}_{1} \cup \ldots \cup \mathcal{Y}_{\max },\left(X_{1}, \vec{H}^{r \Delta-1}\right)$ is uniform distribution over $\{0,1\}^{n} \times \mathcal{H}^{r \Delta-1}$. We also naturally extend the definition of collision probability conditioned on $\mathcal{E}_{k}$ and $\mathcal{S}_{k}$. For example,

$$
\begin{gathered}
\mathrm{CP}\left(Y_{k} \wedge \mathcal{E}_{k} \wedge \mathcal{S}_{k} \mid \vec{H}^{r \Delta-1}\right) \stackrel{\text { def }}{=} \mathbb{E}_{\vec{h}^{r \Delta-1} \leftarrow \vec{H}^{r \Delta-1}}\left[\sum_{y} \operatorname{Pr}\left[f^{k}\left(X_{1}, \vec{H}^{r \Delta-1}\right)=y \wedge \mathcal{E}_{k} \wedge \mathcal{S}_{k} \mid \vec{H}^{r \Delta-1}=\vec{h}^{r \Delta-1}\right]^{2}\right], \\
\mathrm{CP}\left(Y_{k}, \vec{H}^{r \Delta-1} \mid \mathcal{E}_{k} \wedge \mathcal{S}_{k}\right) \stackrel{\text { def }}{=} \sum_{\left(y, \vec{h}^{r \Delta-1}\right)} \operatorname{Pr}\left[\left(f^{k}\left(X_{1}, \vec{H}^{r \Delta-1}\right), \vec{H}^{r \Delta-1}\right)=\left(y, \vec{h}^{r \Delta-1}\right) \mid \mathcal{E}_{k} \wedge \mathcal{S}_{k}\right]^{2} .
\end{gathered}
$$

Claim 4.1 For any $n \in \mathbb{N}$, let $\mathcal{S}_{k}$ and $\mathcal{E}_{k}$ be as defined in Definition 4.1, assume that $f$ is weakly-regular (with $c, \epsilon$ and $\max$ defined as in (1) and (2)). Then, it holds that

$$
\begin{gathered}
\forall k \in[r \Delta]: \operatorname{Pr}\left[\mathcal{S}_{k}\right] \geq 1-k \epsilon, \quad \operatorname{Pr}\left[\mathcal{E}_{k}\right] \geq n^{-c}, \quad \operatorname{Pr}\left[\mathcal{E}_{k} \wedge \mathcal{S}_{k}\right] \geq n^{-c} / 2 \\
\forall k \in \mathbb{N}: \operatorname{Pr}\left[\mathcal{E}_{k+1} \vee \mathcal{E}_{k+2} \vee \ldots \vee \mathcal{E}_{k+\Delta}\right] \geq 1-\exp ^{\Delta / n^{2 c}} \geq 1-n^{-\alpha} \\
\forall k \in[r \Delta]: \operatorname{CP}\left(Y_{k} \wedge \mathcal{E}_{k} \wedge \mathcal{S}_{k} \mid \vec{H}^{r \Delta-1}\right) \leq r \Delta \cdot 2^{\max -n+1}, \text { where } Y_{k}=f^{k}\left(X_{1}, \vec{H}^{r \Delta-1}\right) .
\end{gathered}
$$


Proof. We have that $x_{1}, x_{2}=h_{1}\left(y_{1}\right), \ldots, x_{r \Delta}=h_{r \Delta-1}\left(y_{r \Delta-1}\right)$ are all i.i.d. to $U_{n}$ due to the universality of $\mathcal{H}$. This implies that $\operatorname{Pr}\left[y_{i} \in \mathcal{Y}_{[\max ]}\right] \geq 1-\epsilon$ for every $i \in[k]$ independently, and that $\mathcal{E}_{1}, \ldots$ and $\mathcal{E}_{r \Delta}$ are i.i.d. events with probability at least $n^{-c}$. The former further implies

$$
\operatorname{Pr}\left[\mathcal{S}_{k}\right] \geq(1-\epsilon)^{k} \geq 1-k \cdot \epsilon
$$

where the second inequality is due to Fact A.2. Thus, we complete the proof for (12) by

$$
\operatorname{Pr}\left[\mathcal{E}_{k} \wedge \mathcal{S}_{k}\right] \geq \operatorname{Pr}\left[\mathcal{E}_{k}\right]-\operatorname{Pr}\left[\neg \mathcal{S}_{k}\right] \geq n^{-c}-k \cdot \epsilon \geq n^{-c} / 2
$$

For every $k \in \mathbb{N}, i \in[\Delta]$, define $\zeta_{k+i}=1$ iff $\mathcal{E}_{k+i}$ occurs (and $\zeta_{k+i}=0$ otherwise). It follows by a Chernoff-Hoeffding bound that

$$
\forall k \in \mathbb{N}: \operatorname{Pr}\left[\left(\neg \mathcal{E}_{k+1}\right) \wedge \ldots \wedge\left(\neg \mathcal{E}_{k+\Delta}\right)\right]=\operatorname{Pr}\left[\sum_{i=1}^{\Delta} \zeta_{k+i}=0\right] \leq \exp ^{-\Delta / n^{2 c}} \leq n^{-\alpha}
$$

which yields (13) by taking a negation. For the collision probability in (14), we consider two instances of the randomized iterate seeded with independent $x_{1}$ and $x_{1}^{\prime}$ and a common random $\vec{h}^{r \Delta-1}$ and thus:

$$
\begin{aligned}
& \operatorname{CP}\left(Y_{k} \wedge \mathcal{E}_{k} \wedge \mathcal{S}_{k} \mid \vec{H}^{r \Delta-1}\right) \leq \mathrm{CP}\left(Y_{k} \wedge \mathcal{S}_{k} \mid \vec{H}^{r \Delta-1}\right) \\
& \leq \quad \operatorname{Pr}_{x_{1}, x_{1}^{\prime} \Phi_{\{0,1\}^{n}}}\left[f\left(x_{1}\right)=f\left(x_{1}^{\prime}\right) \in \mathcal{Y}_{[\text {max }]}\right]+\sum_{t=2}^{k}(\underbrace{\operatorname{Pr}}_{y_{t-1} \neq y_{t-1}^{\prime}, h_{t-1} \stackrel{\Phi}{\leftarrow} \mathcal{H}}\left[f\left(x_{t}\right)=f\left(x_{t}^{\prime}\right) \in \mathcal{Y}_{[\text {max }]}\right]) \\
& \leq r \Delta \sum_{y \in \mathcal{Y}_{[\max ]}} \operatorname{Pr}\left[f\left(U_{n}\right)=y\right]^{2} \leq r \Delta \sum_{i=1}^{\max } \sum_{y \in \mathcal{Y}_{i}} \operatorname{Pr}\left[f\left(U_{n}\right)=y\right] \cdot 2^{i-n}=r \Delta \sum_{i=1}^{\max } \operatorname{Pr}\left[f\left(U_{n}\right) \in \mathcal{Y}_{i}\right] \cdot 2^{i-n} \\
& \leq r \Delta \cdot 2^{\max -n}\left(1+2^{-1}+\ldots+2^{-(\max -1)}\right) \leq r \Delta \cdot 2^{\max -n+1} .
\end{aligned}
$$

where we omit $\mathcal{E}_{k}$ in the first inequality (since we are considering upper bound), the second inequality is due to that the collision probability is upper bounded by the sum of events that the first collision occurs on points $y_{1}, y_{2}, \ldots, y_{k} \in \mathcal{Y}_{[\max ]}$ respectively, and the third inequality follows from the pairwise independence of $\mathcal{H}$ so that $x_{1}, x_{1}^{\prime}, \ldots, x_{k}$ and $x_{k}^{\prime}$ are i.i.d. to $U_{n}$.

Lemma 4.2 For any $n \in \mathbb{N}$, with the same assumptions and notations as in Theorem 4.1, Definition 2.4 and Definition 4.1, and let $j^{*} \in[r], \mathrm{A}, \varepsilon_{\mathrm{A}}$ be as assumed in (11). Then, there exists $i^{*} \in[\Delta]$ such that

$$
\operatorname{Pr}\left[\mathrm{A}\left(Y_{j^{*} \cdot \Delta}, \vec{H}^{r \Delta-1}\right)=X_{1+\left(j^{*}-1\right) \Delta} \wedge \mathcal{E}_{\left(j^{*}-1\right) \Delta+i^{*}} \wedge \mathcal{S}_{\left(j^{*}-1\right) \Delta+i^{*}}\right] \geq \varepsilon_{\mathrm{A}} / 2 \Delta .
$$

Proof. For notational convenience use shorthand $\mathcal{C}$ for the event $\mathrm{A}\left(Y_{j^{*} \cdot \Delta}, \vec{H}^{r \Delta-1}\right)=X_{1+\left(j^{*}-1\right) \Delta}$. Then,

$$
\begin{array}{r}
\sum_{i=1}^{\Delta} \operatorname{Pr}\left[\mathcal{C} \wedge \mathcal{E}_{\left(j^{*}-1\right) \Delta+i} \wedge \mathcal{S}_{\left(j^{*}-1\right) \Delta+i}\right] \geq \sum_{i=1}^{\Delta} \operatorname{Pr}\left[\mathcal{C} \wedge \mathcal{E}_{\left(j^{*}-1\right) \Delta+i} \wedge \mathcal{S}_{r \Delta}\right] \geq \operatorname{Pr}\left[\mathcal{C} \wedge \mathcal{S}_{r \Delta} \wedge\left(\bigvee_{i=1}^{\Delta} \mathcal{E}_{\left(j^{*}-1\right) \Delta+i}\right)\right] \\
\geq \operatorname{Pr}[\mathcal{C}]-\operatorname{Pr}\left[\neg \mathcal{S}_{r \Delta}\right]-\operatorname{Pr}\left[\neg\left(\bigvee_{i=1}^{\Delta} \mathcal{E}_{\left(j^{*}-1\right) \Delta+i}\right)\right] \geq \varepsilon_{\mathrm{A}}-r \Delta \cdot \epsilon-n^{-\alpha} \geq \varepsilon_{\mathrm{A}} / 2,
\end{array}
$$

where the first inequality is due to $\mathcal{S}_{r \Delta} \subseteq \mathcal{S}_{\kappa}$ for any $\kappa \leq r \Delta$, the second inequality is the union bound, and the fourth follows from (12) and (13). We recall that $\epsilon$ and $n^{-\alpha}$ are both negligible in $n$. Thus, there exists $i^{*}$ (that satisfies (15)) by an averaging argument.

The Intuition For $\mathrm{M}^{\mathrm{A}}$. Lemma 4.2 states that there exist some $i^{*}$ and $j^{*}$ conditioned on which A inverts the iterate with non-negligible probability. If we knew which $i^{*}$ and $j^{*}$, then we simply replace 
$y_{\left(j^{*}-1\right) \Delta+i^{*}}$ with $f\left(U_{n}\right)$, simulate the iterate for the rest iterations and invoke A to invert $f$. Although the distribution after the replacement will not be identical to the original one, we use Lemma 3.1 to argue that the Rényi entropy deficiency is small enough and thus the inverting probability will not blow up by more than a polynomial factor. However, we actually do not know the values of $i^{*}$ and $j^{*}$, so we need to randomly sample $i$ and $j$ over $[\Delta],[r]$ respectively. This yields $\mathrm{M}^{\mathrm{A}}$ as defined in Algorithm 1.

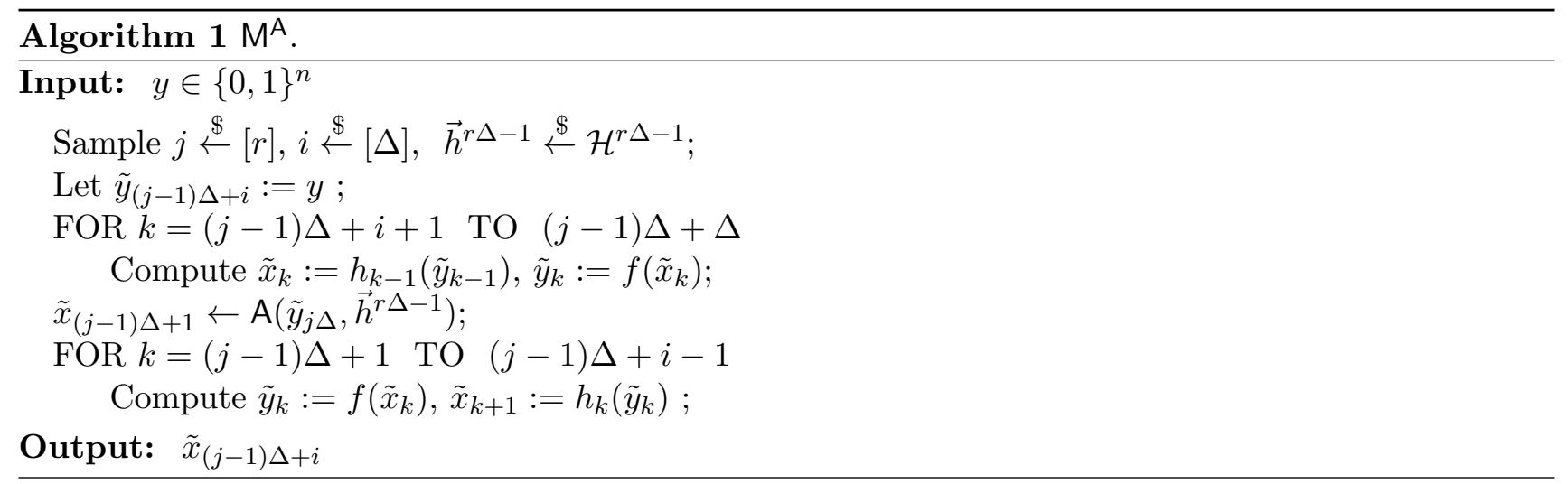

Lemma $4.3\left(\mathrm{M}^{\mathrm{A}}\right.$ inverts $\left.f\right)$ For any $n \in \mathbb{N}$, let $\mathrm{A}$ be as assumed in Lemma 4.2 and let $\mathrm{M}^{\mathrm{A}}$ be as defined in Algorithm 1. Then, it holds that

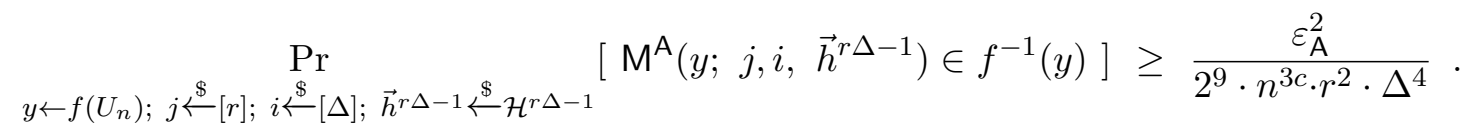

Proof. We know by Lemma 4.2 that there exist $j^{*} \in[r]$ and $i^{*} \in[\Delta]$ satisfying (15), which implies

$$
\begin{aligned}
& \operatorname{Pr}\left[\mathrm{M}^{\mathrm{A}}\left(Y_{(j-1) \Delta+i} ; j, i, \vec{H}^{r \Delta-1}\right) \in f^{-1}\left(Y_{(j-1) \Delta+i}\right) \mid(j, i)=\left(j^{*}, i^{*}\right) \wedge \mathcal{E}_{(j-1) \Delta+i} \wedge \mathcal{S}_{(j-1) \Delta+i}\right] \\
& \geq \operatorname{Pr}\left[\mathrm{A}\left(Y_{j^{*} \cdot \Delta}, \vec{H}^{r \Delta-1}\right)=X_{1+\left(j^{*}-1\right) \Delta} \mid \mathcal{E}_{\left(j^{*}-1\right) \Delta+i^{*}} \wedge \mathcal{S}_{\left(j^{*}-1\right) \Delta+i^{*}}\right] \\
& \geq \operatorname{Pr}\left[\mathrm{A}\left(Y_{j^{*} \cdot \Delta}, \vec{H}^{r \Delta-1}\right)=X_{1+\left(j^{*}-1\right) \Delta} \wedge \mathcal{E}_{\left(j^{*}-1\right) \Delta+i^{*}} \wedge \mathcal{S}_{\left(j^{*}-1\right) \Delta+i^{*}}\right] \geq \varepsilon_{\mathrm{A}} / 2 \Delta,
\end{aligned}
$$

where the second inequality, in abstract form, is $\operatorname{Pr}\left[\mathcal{E}_{a} \mid \mathcal{E}_{b}\right] \geq \operatorname{Pr}\left[\mathcal{E}_{a} \mid \mathcal{E}_{b}\right] \cdot \operatorname{Pr}\left[\mathcal{E}_{b}\right]=\operatorname{Pr}\left[\mathcal{E}_{a} \wedge \mathcal{E}_{b}\right]$. The above is not exactly what we need as conditioned on $\mathcal{E}_{\left(j^{*}-1\right) \Delta+i^{*}} \wedge \mathcal{S}_{\left(j^{*}-1\right) \Delta+i^{*}}$, the random variable $\left(Y_{\left(j^{*}-1\right) \Delta+i^{*}}, \vec{H}^{r \Delta-1}\right)$ is not uniform over $\mathcal{Y}_{\max } \times \mathcal{H}^{r \Delta-1}$. However, we show below that it has nearly full Rényi entropy over $\mathcal{Y}_{\max } \times \mathcal{H}^{r \Delta-1}$

$$
\begin{aligned}
& \operatorname{CP}\left(\left(Y_{\left(j^{*}-1\right) \Delta+i^{*}}, \vec{H}^{r \Delta-1}\right) \mid \mathcal{E}_{\left(j^{*}-1\right) \Delta+i^{*}} \wedge \mathcal{S}_{\left(j^{*}-1\right) \Delta+i^{*}}\right) \\
= & \operatorname{CP}\left(\left(Y_{\left(j^{*}-1\right) \Delta+i^{*}}, \vec{H}^{r \Delta-1}\right) \wedge \mathcal{E}_{\left(j^{*}-1\right) \Delta+i^{*}} \wedge \mathcal{S}_{\left(j^{*}-1\right) \Delta+i^{*}}\right) / \operatorname{Pr}\left[\mathcal{E}_{\left(j^{*}-1\right) \Delta+i^{*}} \wedge \mathcal{S}_{\left(j^{*}-1\right) \Delta+i^{*}}\right]^{2} \\
\leq & \operatorname{CP}\left(Y_{\left(j^{*}-1\right) \Delta+i^{*}} \wedge \mathcal{E}_{\left(j^{*}-1\right) \Delta+i^{*}} \wedge \mathcal{S}_{\left(j^{*}-1\right) \Delta+i^{*}} \mid \vec{H}^{r \Delta-1}\right) \frac{1}{\left(n^{-2 c} / 4\right) \cdot|\mathcal{H}|^{r \Delta-1}} \\
\leq & \frac{r \Delta \cdot 2^{\max -n+1}}{\left(n^{-2 c} / 4\right) \cdot|\mathcal{H}|^{r \Delta-1}}=\frac{8 r \Delta \cdot n^{2 c}}{2^{n-\max } \cdot|\mathcal{H}|^{r \Delta-1}},
\end{aligned}
$$

where the first equality follows from Fact A.1 (see Appendix A) and the two inequalities are by (12) and (14) respectively. Taking a negative logarithm, we get

$$
\mathbf{H}_{2}\left(\left(Y_{\left(j^{*}-1\right) \Delta+i^{*}}, \vec{H}^{r \Delta-1}\right) \mid \mathcal{E}_{\left(j^{*}-1\right) \Delta+i^{*}} \wedge \mathcal{S}_{\left(j^{*}-1\right) \Delta+i^{*}}\right) \geq(n-\max +(r \Delta-1) \log |\mathcal{H}|+1)-e,
$$


where entropy deficiency $e \leq 2 c \cdot \log n+\log r+\log \Delta+4$. This is due to that the uniform distribution over $\mathcal{Y}_{\max } \times \mathcal{H}^{r \Delta-1}$ has full entropy

$$
\mathbf{H}_{2}\left(\left(U_{\mathcal{Y}_{\max }}, \vec{H}^{r \Delta-1}\right)\right) \leq \log \left(\frac{1}{2^{-n+\max -1}} \cdot|\mathcal{H}|^{r \Delta-1}\right)=n-\max +(r \Delta-1) \log |\mathcal{H}|+1 .
$$

To apply Lemma 3.1 , let $\mathcal{W}=\mathcal{Y}_{\max } \times \mathcal{H}^{r \Delta-1}, \mathcal{Z}=\emptyset$, let $W$ be $\left(Y_{\left(j^{*}-1\right) \Delta+i^{*}}, \vec{H}^{r \Delta-1}\right)$ conditioned on $\mathcal{E}_{\left(j^{*}-1\right) \Delta+i^{*}}$ and $\mathcal{S}_{\left(j^{*}-1\right) \Delta+i^{*}}$, and define

$$
\operatorname{Adv}\left(y, \vec{h}^{r \Delta-1}\right) \stackrel{\text { def }}{=} \begin{cases}1, & \text { if } \mathrm{M}^{\mathrm{A}}\left(y ; j^{*}, i^{*}, \vec{h}^{r \Delta-1}\right) \in f^{-1}(y) \\ 0, & \text { if } \mathrm{M}^{\mathrm{A}}\left(y ; j^{*}, i^{*}, \vec{h}^{r \Delta-1}\right) \notin f^{-1}(y)\end{cases}
$$

Let $\mathcal{C}_{j^{*} i^{*} \text { max }}$ denote the event that $(j, i)=\left(j^{*}, i^{*}\right) \wedge f\left(U_{n}\right) \in \mathcal{Y}_{\max }$, and we thus have

$$
\begin{aligned}
& \operatorname{Pr}\left[\mathrm{M}^{\mathrm{A}}\left(f\left(U_{n}\right) ; j, i, \vec{H}^{r \Delta-1}\right) \in f^{-1}\left(f\left(U_{n}\right)\right)\right] \\
\geq & \operatorname{Pr}\left[\mathcal{C}_{j^{*} i^{*} \max }\right] \cdot \operatorname{Pr}\left[\mathrm{M}^{\mathrm{A}}\left(f\left(U_{n}\right) ; j, i, \vec{H}^{r \Delta-1}\right) \in f^{-1}\left(f\left(U_{n}\right)\right) \mid \mathcal{C}_{j^{*} i^{*} \max }\right] \\
\geq & \left(1 / r \Delta n^{c}\right) \cdot \mathbb{E}\left[\operatorname{Adv}\left(U_{\mathcal{Y}_{\text {max }}}, \vec{H}^{r \Delta-1}\right)\right] / 2 \\
\geq & \left(1 / r \Delta n^{c}\right) \cdot \frac{\mathbb{E}\left[\operatorname{Adv}\left(Y_{\left(j^{*}-1\right) \Delta+i^{*}}, \vec{H}^{r \Delta-1}\right) \mid \mathcal{E}_{\left(j^{*}-1\right) \Delta+i^{*}} \wedge \mathcal{S}_{\left(j^{*}-1\right) \Delta+i^{*}}\right]^{2}}{2^{e+3}} \\
\geq & \left(1 / r \Delta n^{c}\right) \cdot \frac{\varepsilon_{\mathrm{A}}^{2} / 4 \Delta^{2}}{2^{7} \cdot n^{2 c} r \cdot \Delta}=\frac{\varepsilon_{\mathrm{A}}^{2}}{2^{9} \cdot n^{3 c} \cdot r^{2} \cdot \Delta^{4}},
\end{aligned}
$$

where the second inequality is due to Claim A.1 (i.e., conditioned on $f\left(U_{n}\right) \in \mathcal{Y}_{\text {max }}$ random variable $f\left(U_{n}\right)$ can be loosely regarded as $\left.U_{\mathcal{Y}_{\max }}\right)$, and the third inequality follows from Lemma 3.1.

\subsection{The Derandomized Version: A PRG with Seed Length $O(n \cdot \log n)$}

The derandomized version uses a bounded-space generator to expand an $O(n \cdot \log n)$-bit $u$ into a long string over $\mathcal{H}^{r \Delta-1}$ (rather than sampling a random element over it).

Theorem 4.2 (the derandomized version) For $n, k \in \mathbb{N}$, let $f, c, \mathcal{H}, \mathcal{H}_{c}, f^{k}, \alpha=\alpha(n), \Delta=\Delta(n)$ and $r=r(n)$ be as assumed in Theorem 4.1, let $g$ be as defined in (9), let

$$
B S G:\{0,1\}^{q(n) \in O(n \cdot \log n)} \rightarrow\{0,1\}^{\left(\alpha \cdot n^{2 c+1}-1\right) \cdot \log |\mathcal{H}|}
$$

be a bounded-space generator that $2^{-2 n}$-fools every $\left(2 n+1, \alpha \cdot n^{2 c+1}, \log |\mathcal{H}|\right)-L B P$ (see Footnote 7). Then, the function $g^{\prime}:\{0,1\}^{n} \times\{0,1\}^{q} \times \mathcal{H}_{c} \rightarrow\{0,1\}^{2 n} \times\{0,1\}^{q} \times \mathcal{H}_{c}$ defined as

$$
g^{\prime}\left(x_{1}, u, h_{c}\right)=g\left(x_{1}, B S G(u), h_{c}\right)
$$

is a pseudorandom generator.

Similar to the randomized version, it suffices to show Lemma 4.4 (the counterpart of Lemma 4.1).

Lemma 4.4 For the same assumptions as stated in Lemma 4.1, we have that for every $j \in[r]$, and for every PPT A' of running time $T(n)-n^{O(1)}$ (for some universal constant $O(1)$ ) it holds that

$$
x_{1} \stackrel{\$}{\leftarrow}\{0,1\}^{n}, u \stackrel{\$}{\stackrel{P r}{\{}\{0,1\}^{q},{\overrightarrow{h^{\prime}}}^{r \Delta-1}:=B S G(u)}\left[\mathrm{A}^{\prime}\left(y_{j \cdot \Delta}^{\prime}, u\right)=x_{1+(j-1) \Delta}^{\prime}\right] \in O\left(n^{3 c / 2} \cdot r \cdot \Delta^{2} \cdot \sqrt{\varepsilon}\right) .
$$

where $y_{k}^{\prime}=f^{k}\left(x_{1},{\overrightarrow{h^{\prime}}}^{r \Delta-1}\right)$ and $x_{k+1}^{\prime}=h_{k}^{\prime}\left(y_{k}^{\prime}\right)$ for $k \in \mathbb{N}$. 
The proof of Lemma 4.4 follows the steps of that of Lemma 4.1. We define events $\mathcal{S}_{k}^{\prime}$ and $\mathcal{E}_{k}^{\prime}$ in Definition 4.2 (the analogues of $\mathcal{S}_{k}$ and $\mathcal{E}_{k}$ ). Although the events (e.g., $\mathcal{E}_{1}^{\prime}, \ldots, \mathcal{E}_{k}^{\prime}$ ) are not independent due to short of randomness, we still have (18), (19) and (20) below. We defer their proofs to Appendix A due to lack of space, where for every inequality we define an LBP and argue that the advantage of the LBP on $\vec{H}^{r \Delta-1}$ and $B S G\left(U_{q}\right)$ is bounded by $2^{-2 n}$ and thus (18), (19) and (20) follow from their respective counterparts (12), (13) and (14) by adding an additive term $2^{-2 n}$.

Definition 4.2 (events $\mathcal{S}_{k}^{\prime}$ and $\mathcal{E}_{k}^{\prime}$ ) For any $n \in \mathbb{N}$ and any $k \leq r \Delta$, define events

$$
\begin{gathered}
\mathcal{S}_{k}^{\prime} \stackrel{\text { def }}{=}\left(\left(X_{1}, U_{q}\right) \in\left\{\left(x_{1}, u\right): \forall t \in[k] \text { satisfies } y_{t}^{\prime} \in \mathcal{Y}_{[\max ]}, \text { where } y_{t}^{\prime}=f^{t}\left(x_{1}, B S G(u)\right)\right\}\right) \\
\mathcal{E}_{k}^{\prime} \stackrel{\text { def }}{=}\left(\left(X_{1}, U_{q}\right) \in\left\{\left(x_{1}, u\right): y_{k}^{\prime} \in \mathcal{Y}_{\max }, \text { where } y_{k}^{\prime}=f^{k}\left(x_{1}, B S G(u)\right)\right\}\right)
\end{gathered}
$$

where $\left(X_{1}, U_{q}\right)$ is uniform distribution over $\{0,1\}^{n} \times\{0,1\}^{q}$. We refer to Definition B. 1 in Appendix $B$ for the definitions of the collision probabilities in the following proofs.

$$
\begin{gathered}
\forall k \in[r \Delta]: \operatorname{Pr}\left[\mathcal{S}_{k}^{\prime}\right] \geq 1-k \epsilon-2^{-2 n}, \quad \operatorname{Pr}\left[\mathcal{E}_{k}^{\prime}\right] \geq n^{-c}-2^{-2 n}, \quad \operatorname{Pr}\left[\mathcal{E}_{k}^{\prime} \wedge \mathcal{S}_{k}^{\prime}\right] \geq n^{-c} / 2 \\
\forall k \in[(r-1) \Delta]: \operatorname{Pr}\left[\mathcal{E}_{k+1}^{\prime} \vee \mathcal{E}_{k+2}^{\prime} \vee \ldots \vee \mathcal{E}_{k+\Delta}^{\prime}\right] \geq 1-n^{-\alpha}-2^{-2 n} \\
\forall k \in[r \Delta]: \operatorname{CP}\left(Y_{k}^{\prime} \wedge \mathcal{E}_{k}^{\prime} \wedge \mathcal{S}_{k}^{\prime} \mid U_{q}\right) \leq(r \Delta+1) \cdot 2^{\text {max }-n+1}, \quad \text { where } Y_{k}^{\prime}=f^{k}\left(X_{1}, B S G\left(U_{q}\right)\right)
\end{gathered}
$$

Proof SKetch of Lemma 4.4. Assume towards a contradiction that for some non-negligible $\varepsilon_{\mathrm{A}^{\prime}}=$ $\varepsilon_{\mathrm{A}^{\prime}}(n)$ that

$$
\exists j^{*} \in[r], \exists \mathrm{PPT} \mathrm{A}^{\prime}: \operatorname{Pr}\left[\mathrm{A}^{\prime}\left(Y_{j^{*} \cdot \Delta}^{\prime}, U_{q}\right)=X_{1+\left(j^{*}-1\right) \Delta}^{\prime}\right] \geq \varepsilon_{\mathrm{A}^{\prime}}
$$

where for $k \in[r \Delta]$ we use notations $\vec{H}^{\prime r \Delta-1}=B S G\left(U_{q}\right), Y_{k}^{\prime}=f^{k}\left(X_{1}, \vec{H}^{\prime r \Delta-1}\right)$ and $X_{k+1}^{\prime}=H_{k}^{\prime}\left(Y_{k}^{\prime}\right)$. Then, we define $\mathrm{M}^{\mathrm{A}^{\prime}}$ that inverts $f$ with the following probability. Since $\mathrm{M}^{\mathrm{A}^{\prime}}$ is quite similar to its analogue $\mathrm{M}^{\mathrm{A}}$ we state it as Algorithm 2 in Appendix B.

$$
\operatorname{Pr}_{y \leftarrow f\left(U_{n}\right) ; j \stackrel{\$}{\leftarrow}[r] ; i \stackrel{\$}{\leftarrow}[\Delta] ; u \stackrel{\$}{\leftarrow}\{0,1\}^{q}}\left[\mathrm{M}^{\mathrm{A}^{\prime}}(y ; j, i, u) \in f^{-1}(y)\right] \in \Omega\left(\frac{\varepsilon_{\mathrm{A}^{\prime}}^{2}}{n^{3 c} \cdot r^{2} \cdot \Delta^{4}}\right),
$$

which is a contradiction to the one-way-ness of $f$ and thus concludes Lemma 4.4.

Proof sketch of $(22)$. Denote by $\mathcal{C}^{\prime}$ the event $\mathrm{A}^{\prime}\left(Y_{j^{*} \cdot \Delta}^{\prime}, U_{q}\right)=X_{1+\left(j^{*}-1\right) \Delta}^{\prime}$. Then,

$$
\begin{aligned}
\sum_{i=1}^{\Delta} \operatorname{Pr}\left[\mathcal{C}^{\prime}\right. & \left.\wedge \mathcal{E}_{\left(j^{*}-1\right) \Delta+i}^{\prime} \wedge \mathcal{S}_{\left(j^{*}-1\right) \Delta+i}^{\prime}\right] \geq \sum_{i=1}^{\Delta} \operatorname{Pr}\left[\mathcal{C}^{\prime} \wedge \mathcal{E}_{\left(j^{*}-1\right) \Delta+i}^{\prime} \wedge \mathcal{S}_{r \Delta}^{\prime}\right] \geq \operatorname{Pr}\left[\mathcal{C}^{\prime} \wedge \mathcal{S}_{r \Delta}^{\prime} \wedge\left(\bigvee_{i=1}^{\Delta} \mathcal{E}_{\left(j^{*}-1\right) \Delta+i}^{\prime}\right)\right] \\
& \geq \operatorname{Pr}\left[\mathcal{C}^{\prime}\right]-\operatorname{Pr}\left[\neg \mathcal{S}_{r \Delta}^{\prime}\right]-\operatorname{Pr}\left[\neg\left(\bigvee_{i=1}^{\Delta} \mathcal{E}_{\left(j^{*}-1\right) \Delta+i}^{\prime}\right)\right] \geq \varepsilon_{A^{\prime}}-r \Delta \cdot \epsilon-n^{-\alpha}-2^{-2 n+1} \geq \varepsilon_{A^{\prime}} / 2
\end{aligned}
$$

where the first three inequalities are similar to analogues in the proof of Lemma 4.2 and the fourth inequality is due to (18) and (19). Thus, by averaging we have that

$\exists j^{*} \in[r], \exists i^{*} \in[\Delta], \exists \mathrm{PPT} \mathrm{A}^{\prime}: \operatorname{Pr}\left[\mathrm{A}^{\prime}\left(Y_{j^{*} \cdot \Delta}^{\prime}, U_{q}\right)=X_{1+\left(j^{*}-1\right) \Delta}^{\prime} \wedge \mathcal{E}_{\left(j^{*}-1\right) \Delta+i^{*}}^{\prime} \wedge \mathcal{S}_{\left(j^{*}-1\right) \Delta+i^{*}}^{\prime}\right] \geq \varepsilon_{\mathrm{A}^{\prime}} / 2 \Delta$.

The proofs below follow the steps of Lemma 4.3. We have that (proof of (23) given in Appendix A)

$$
\mathbf{H}_{2}\left(\left(Y_{\left(j^{*}-1\right) \Delta+i^{*}}^{\prime}, U_{q}\right) \mid \mathcal{E}_{\left(j^{*}-1\right) \Delta+i^{*}}^{\prime} \wedge \mathcal{S}_{\left(j^{*}-1\right) \Delta+i^{*}}^{\prime}\right) \geq \mathbf{H}_{2}\left(U_{\mathcal{Y}_{\max }}, U_{q}\right)-e,
$$


where entropy deficiency $e \leq 2 c \cdot \log n+\log r+\log \Delta+5$. Finally, let $\mathcal{W}=\mathcal{Y}_{\max } \times\{0,1\}^{q}, \mathcal{Z}=\emptyset$, let $W$ be $\left(Y_{\left(j^{*}-1\right) \Delta+i^{*}}^{\prime}, U_{q}\right)$ conditioned on $\mathcal{E}_{\left(j^{*}-1\right) \Delta+i^{*}}^{\prime}$ and $\mathcal{S}_{\left(j^{*}-1\right) \Delta+i^{*}}^{\prime}$, and define

$$
\operatorname{Adv}(y, u) \stackrel{\text { def }}{=}\left\{\begin{array}{l}
1, \text { if } \mathrm{M}^{\mathrm{A}^{\prime}}\left(y ; j^{*}, i^{*}, u\right) \in f^{-1}(y) \\
0, \text { if } \mathrm{M}^{\mathrm{A}^{\prime}}\left(y ; j^{*}, i^{*}, u\right) \notin f^{-1}(y)
\end{array}\right.
$$

Let $\mathcal{C}_{j^{*} i^{*} \text { max }}$ denote the event that $(j, i)=\left(j^{*}, i^{*}\right) \wedge f\left(U_{n}\right) \in \mathcal{Y}_{\max }$, and we thus have

$$
\begin{aligned}
& \operatorname{Pr}\left[\mathrm{M}^{\mathrm{A}^{\prime}}\left(f\left(U_{n}\right) ; j, i, U_{q}\right) \in f^{-1}\left(f\left(U_{n}\right)\right)\right] \\
\geq & \operatorname{Pr}\left[\mathcal{C}_{j^{*} i^{*} \max }\right] \cdot \operatorname{Pr}\left[\mathrm{M}^{\mathrm{A}^{\prime}}\left(f\left(U_{n}\right) ; j, i, U_{q}\right) \in f^{-1}\left(f\left(U_{n}\right)\right) \mid \mathcal{C}_{j^{*} i^{*} \max }\right] \\
\geq & \left(1 / r \Delta n^{c}\right) \cdot \mathbb{E}\left[\operatorname { A d v } \left(U_{\left.\left.\mathcal{Y}_{\max }, U_{q}\right)\right] / 2}\right.\right. \\
\geq & \left(1 / r \Delta n^{c}\right) \cdot \frac{\mathbb{E}\left[\operatorname{Adv}\left(Y_{\left(j^{*}-1\right) \Delta+i^{*}}^{\prime}, U_{q}\right) \mid \mathcal{E}_{\left(j^{*}-1\right) \Delta+i^{*}}^{\prime} \wedge \mathcal{S}_{\left(j^{*}-1\right) \Delta+i^{*}}^{\prime}\right]^{2}}{2^{e+3}} \\
\geq & \left(1 / r \Delta n^{c}\right) \cdot \frac{\operatorname{Pr}\left[\mathrm{A}^{\prime}\left(Y_{j^{*} \cdot \Delta}^{\prime}, U_{q}\right)=X_{1+\left(j^{*}-1\right) \Delta}^{\prime} \wedge \mathcal{E}_{\left(j^{*}-1\right) \Delta+i^{*}}^{\prime} \wedge \mathcal{S}_{\left(j^{*}-1\right) \Delta+i^{*}}^{\prime}\right]^{2}}{2^{e+3}} \\
\geq & \left(1 / r \Delta n^{c}\right) \cdot \frac{\varepsilon_{\mathrm{A}^{\prime}}^{2} / 4 \Delta^{2}}{2^{8} \cdot n^{2 c} r \cdot \Delta}=\frac{\varepsilon_{\mathrm{A}^{\prime}}^{2}}{2^{10} \cdot n^{3 c} \cdot r^{2} \cdot \Delta^{4}} \cdot
\end{aligned}
$$

where the inequalities follow the same order as their analogues in the proof of Lemma 4.3.

\section{Acknowledgement}

This research work was supported by the National Basic Research Program of China (Grant 2013CB338004). $\mathrm{Yu} \mathrm{Yu}$ was supported by the National Natural Science Foundation of China Grant (Nos. 61472249, 61103221). Dawu Gu was supported by the National Natural Science Foundation of China Grant (Nos. 61472250, 61402286), the Doctoral Fund of Ministry of Education of China (No. 20120073110094) and the Innovation Program by Shanghai Municipal Science and Technology Commission (No. 14511100300). Xiangxue Li was supported by the National Natural Science Foundation of China (Nos. 61472472, 61272536, 61172085) and Science and Technology Commission of Shanghai Municipality (Grant 13JC1403500).

Jian Weng was supported by the National Science Foundation of China (Nos. 61272413, 61133014, 61472165), the Fok Ying Tung Education Foundation (No. 131066), the Program for New Century Excellent Talents in University (No. NCET-12-0680), and the Research Fund for the Doctoral Program of Higher Education of China (No. 20134401110011).

\section{References}

[1] Boaz Barak, Yevgeniy Dodis, Hugo Krawczyk, Olivier Pereira, Krzysztof Pietrzak, François-Xavier Standaert, and $\mathrm{Yu} Y \mathrm{Yu}$. Leftover hash lemma, revisited. In Phillip Rogaway, editor, CRYPTO, LNCS, pages 1-20. Springer, 2011.

[2] Manuel Blum and Silvio Micali. How to generate cryptographically strong sequences of pseudo random bits. In Proceedings of the 23rd IEEE Symposium on Foundation of Computer Science, pages $112-117,1982$.

[3] Nenad Dedic, Danny Harnik, and Leonid Reyzin. Saving private randomness in one-way functions and pseudorandom generators. In 5th Theory of Cryptography Conference, pages 607-625, 2008.

[4] Yevgeniy Dodis, Krzysztof Pietrzak, and Daniel Wichs. Key derivation without entropy waste. In Proceedings of the 33rd Annual International Conference on the Theory and Applications of Cryptographic Techniques (Eurocrypt 2014), pages 93-110, 2014. 
[5] Yevgeniy Dodis and Yu Yu. Overcoming weak expectations. In Proceedings of the 10th Theory of Cryptography Conference (TCC 2013), pages 1-22, 2013.

[6] Oded Goldreich. Foundations of Cryptography: Basic Tools. Cambridge University Press, 2001.

[7] Oded Goldreich, Hugo Krawczyk, and Michael Luby. On the existence of pseudorandom generators. SIAM Journal on Computing, 22(6):1163-1175, 1993.

[8] Oded Goldreich and Leonid A. Levin. A hard-core predicate for all one-way functions. In D. S. Johnson, editor, Proceedings of the Twenty First Annual ACM Symposium on Theory of Computing, pages 25-32, Seattle, Washington, 15-17 May 1989.

[9] Oded Goldreich, Leonid A. Levin, and Noam Nisan. On constructing 1-1 one-way functions. In Studies in Complexity and Cryptography. Miscellanea on the Interplay between Randomness and Computation, pages 13-25. 2011.

[10] Iftach Haitner, Danny Harnik, and Omer Reingold. On the power of the randomized iterate. In Proceedings of the 26th International Cryptology Conference (CRYPTO 2006), pages 22-40, 2006.

[11] Iftach Haitner, Danny Harnik, and Omer Reingold. On the power of the randomized iterate. SIAM Journal on Computing, 40(6):1486-1528, 2011. draft of full version available at http: //www.cs.tau.ac.il/ iftachh/papers/RandomizedIteate/RandomIterate.pdf.

[12] Iftach Haitner, Omer Reingold, and Salil P. Vadhan. Efficiency improvements in constructing pseudorandom generators from one-way functions. In Proceedings of the 42nd ACM Symposium on the Theory of Computing, pages 437-446, 2010.

[13] Johan Håstad, Russell Impagliazzo, Leonid A. Levin, and Michael Luby. Construction of pseudorandom generator from any one-way function. SIAM Journal on Computing, 28(4):1364-1396, 1999 .

[14] Thomas Holenstein. Pseudorandom generators from one-way functions: A simple construction for any hardness. In Proceedings of the 3rd Theory of Cryptography Conference (TCC 2006), 2006.

[15] Thomas Holenstein and Makrand Sinha. Constructing a pseudorandom generator requires an almost linear Number of calls. In Proceedings of the 53rd IEEE Symposium on Foundation of Computer Science, pages 698-707, 2012.

[16] Russell Impagliazzo, Noam Nisan, and Avi Wigderson. Pseudorandomness for network algorithms. In Proceedings of the 26th ACM Symposium on the Theory of Computing, pages 356-364, 1994.

[17] Noam Nisan. Pseudorandom generators for space-bounded computation. Combinatorica, 12(4):449461, 1992.

[18] Salil P. Vadhan and Colin Jia Zheng. Characterizing pseudoentropy and simplifying pseudorandom generator constructions. In Proceedings of the 44th ACM Symposium on the Theory of Computing, pages $817-836,2012$.

[19] Andrew Chi-Chih Yao. Theory and applications of trapdoor functions (extended abstract). In Proceedings of the 23rd IEEE Symposium on Foundation of Computer Science, pages 80-91, 1982.

[20] $\mathrm{Yu} \mathrm{Yu,} \mathrm{Xiangxue} \mathrm{Li,} \mathrm{and} \mathrm{Jian} \mathrm{Weng.} \mathrm{Pseudorandom} \mathrm{generators} \mathrm{from} \mathrm{regular} \mathrm{one-way} \mathrm{functions:}$ New constructions with improved parameters. In ASIACRYPT, pages 261-279, 2013. 


\section{A Proofs Omitted}

Proof of Lemma 3.3. (6) follows from the assumption that $f$ is a regular function, and the fact that $f\left(U_{n}\right)$ is independent of any other distributions. As for $(7)$, consider running two instances of the iterate seeded with independent $x_{1}$ and $x_{1}^{\prime}$ and a common random $\vec{h}^{k-1}$, the probability of colliding on $y_{k}$ is upper bounded by the sum of the events that the first collision occurs on points $y_{1}, \ldots, y_{k}$ respectively, where $x_{1}, \ldots, x_{k}$ are all i.i.d. to uniform due to the universality of $\mathcal{H}$. It follows that

$$
\mathrm{CP}\left(Y_{k} \mid \vec{H}^{k-1}\right) \leq k \cdot \mathrm{CP}\left(f\left(U_{n}\right)\right)=\frac{k}{\left|f\left(\{0,1\}^{n}\right)\right|} .
$$

We sketch the proof of (8) as below (see [11, Lemma 3.11] for details): consider the following $(2 n, n+$ $1, \log |\mathcal{H}|)$-LBP $M$ for the input $\left(x_{1}, x_{1}^{\prime}\right)$ : the source node is labeled by $\left(y_{1}=f\left(x_{1}\right), y_{1}^{\prime}=f\left(x_{1}^{\prime}\right)\right)$, and being on node labeled by $\left(y_{i}, y_{i}^{\prime}\right)$ at the $i^{\text {th }}$ layer, it takes the current layer input $h_{i} \in \mathcal{H}$, and computes $y_{i+1}:=f\left(h_{i}\left(y_{i}\right)\right), y_{i+1}^{\prime}:=f\left(h_{i}\left(y_{i}^{\prime}\right)\right)$. Finally, $M$ moves to the 1-labeled node if $y_{n+1}=y_{n+1}^{\prime}$ or the 0-labeled node otherwise. Note that the probability that $M$ outputs 1 is equal to that the two iterates (with inputs $x_{1}$ and $x_{1}^{\prime}$ respectively, and using the same hash function $\vec{h}^{n}$ ) collide on $y_{n+1}=y_{n+1}^{\prime}$. As $B S G 2^{-2 n}$-fools every $(2 n, n+1, \log |\mathcal{H}|)$-LBP (including $\left.M\right)$, replacing uniform $\vec{H}^{k-1}$ with $B S G\left(U_{q}\right)$ will not increase the collision probability by more than $2^{-2 n}$, i.e.,

$$
\mathrm{CP}\left(Y_{k}^{\prime} \mid B S G\left(U_{q}\right)\right) \leq \mathrm{CP}\left(Y_{k} \mid \vec{H}^{k-1}\right)+2^{-2 n} \leq \frac{k}{\left|f\left(\{0,1\}^{n}\right)\right|}+2^{-2 n} \leq \frac{k+1}{\left|f\left(\{0,1\}^{n}\right)\right|} .
$$

and it is not hard to see that for any $\vec{h}^{k-1}$ and any $u_{1}, u_{2} \in B S G^{-1}\left(\vec{h}^{k-1}\right)$

$$
\mathrm{CP}\left(Y_{k}^{\prime} \mid U_{q}=u_{1}\right)=\mathrm{CP}\left(Y_{k}^{\prime} \mid U_{q}=u_{2}\right)=\mathrm{CP}\left(Y_{k}^{\prime} \mid B S G\left(U_{q}\right)=\vec{h}^{k-1}\right) .
$$

We complete the proof by

$$
\mathrm{CP}\left(Y_{k}^{\prime} \mid U_{q}\right)=\mathrm{CP}\left(Y_{k}^{\prime} \mid B S G\left(U_{q}\right)\right) \leq \frac{k+1}{\left|f\left(\{0,1\}^{n}\right)\right|} .
$$

Fact A.1 For any $k \in[r \Delta]$, we have

$$
\mathrm{CP}\left(\left(Y_{k}, \vec{H}^{r \Delta-1}\right) \mid \mathcal{E}_{k} \wedge \mathcal{S}_{k}\right)=\frac{\mathrm{CP}\left(\left(Y_{k}, \vec{H}^{r \Delta-1}\right) \wedge \mathcal{E}_{k} \wedge \mathcal{S}_{k}\right)}{\operatorname{Pr}\left[\mathcal{E}_{k} \wedge \mathcal{S}_{k}\right]^{2}}=\frac{\mathrm{CP}\left(Y_{k} \wedge \mathcal{E}_{k} \wedge \mathcal{S}_{k} \mid \vec{H}^{r \Delta-1}\right)}{\operatorname{Pr}\left[\mathcal{E}_{k} \wedge \mathcal{S}_{k}\right]^{2} \cdot|\mathcal{H}|^{r \Delta-1}}
$$

Proof of Fact A.1. We first have that

$$
\begin{aligned}
& \operatorname{CP}\left(\left(Y_{k}, \vec{H}^{r \Delta-1}\right) \mid \mathcal{E}_{k} \wedge \mathcal{S}_{k}\right) \cdot \operatorname{Pr}\left[\mathcal{E}_{k} \wedge \mathcal{S}_{k}\right]^{2} \\
= & \operatorname{Pr}\left[\mathcal{E}_{k} \wedge \mathcal{S}_{k}\right]^{2} \cdot \sum_{\left(y, \vec{h}^{r \Delta-1}\right)} \operatorname{Pr}\left[\left(Y_{k}, \vec{H}^{r \Delta-1}\right)=\left(y, \vec{h}^{r \Delta-1}\right) \mid \mathcal{E}_{k} \wedge \mathcal{S}_{k}\right]^{2} \\
= & \sum_{\left(y, \vec{h}^{r \Delta-1}\right)}\left(\operatorname{Pr}\left[\left(Y_{k}, \vec{H}^{r \Delta-1}\right)=\left(y, \vec{h}^{r \Delta-1}\right) \mid \mathcal{E}_{k} \wedge \mathcal{S}_{k}\right] \cdot \operatorname{Pr}\left[\mathcal{E}_{k} \wedge \mathcal{S}_{k}\right]\right)^{2} \\
= & \sum_{\left(y, \vec{h}^{r \Delta-1}\right)} \operatorname{Pr}\left[\left(Y_{k}, \vec{H}^{r \Delta-1}\right)=\left(y, \vec{h}^{r \Delta-1}\right) \wedge \mathcal{E}_{k} \wedge \mathcal{S}_{k}\right]^{2} \\
= & \operatorname{CP}\left(\left(Y_{k}, \vec{H}^{r \Delta-1}\right) \wedge \mathcal{E}_{k} \wedge \mathcal{S}_{k}\right),
\end{aligned}
$$


and complete the proof by the following

$$
\begin{aligned}
& \frac{\mathrm{CP}\left(Y_{k} \wedge \mathcal{E}_{k} \wedge \mathcal{S}_{k} \mid \vec{H}^{r \Delta-1}\right)}{|\mathcal{H}|^{r \Delta-1}} \\
= & \frac{1}{|\mathcal{H}|^{r \Delta-1}} \cdot \sum_{\vec{h}^{r \Delta-1}} \operatorname{Pr}\left[H^{r \Delta-1}=\vec{h}^{r \Delta-1}\right] \sum_{y} \operatorname{Pr}\left[Y_{k}=y \wedge \mathcal{E}_{k} \wedge \mathcal{S}_{k} \mid H^{r \Delta-1}=\vec{h}^{r \Delta-1}\right]^{2} \\
= & \sum_{\left(y, \vec{h}^{r \Delta-1}\right)}\left(\operatorname{Pr}\left[H^{r \Delta-1}=\vec{h}^{r \Delta-1}\right] \cdot \operatorname{Pr}\left[Y_{k}=y \wedge \mathcal{E}_{k} \wedge \mathcal{S}_{k} \mid H^{r \Delta-1}=\vec{h}^{r \Delta-1}\right]\right)^{2} \\
= & \sum_{\left(y, \vec{h}^{r \Delta-1}\right)} \operatorname{Pr}\left[\left(Y_{k}, H^{r \Delta-1}\right)=\left(y, \vec{h}^{r \Delta-1}\right) \wedge \mathcal{E}_{k} \wedge \mathcal{S}_{k}\right]^{2} \\
= & \operatorname{CP}\left(\left(Y_{k}, \vec{H}^{r \Delta-1}\right) \wedge \mathcal{E}_{k} \wedge \mathcal{S}_{k}\right) .
\end{aligned}
$$

Claim A.1 $\mathbb{E}\left[\operatorname{Adv}\left(f\left(U_{n}\right), \vec{H}^{r \Delta-1}\right) \mid f\left(U_{n}\right) \in \mathcal{Y}_{\max }\right] \geq \mathbb{E}\left[\operatorname{Adv}\left(U_{\mathcal{Y}_{\max }}, \vec{H}^{r \Delta-1}\right)\right] / 2$

Proof. We recall that $f\left(U_{n}\right)$ is independent of $\vec{H}^{r \Delta-1}$.

$$
\begin{aligned}
& \mathbb{E}\left[\operatorname{Adv}\left(f\left(U_{n}\right), \vec{H}^{r \Delta-1}\right) \mid f\left(U_{n}\right) \in \mathcal{Y}_{\text {max }}\right] \\
= & \sum_{\left(y, \vec{h}^{r \Delta-1}\right) \in \mathcal{Y}_{\max } \times \mathcal{H}^{r \Delta-1}} \operatorname{Pr}\left[\vec{H}^{r \Delta-1}=\vec{h}^{r \Delta-1}\right] \cdot \operatorname{Pr}\left[f\left(U_{n}\right)=y \mid f\left(U_{n}\right) \in \mathcal{Y}_{\max }\right] \cdot \operatorname{Adv}\left(y, \vec{h}^{r \Delta-1}\right) \\
\geq & \frac{1}{2} \sum_{\left(y, \vec{h}^{r \Delta-1}\right) \in \mathcal{Y}_{\max } \times \mathcal{H}^{r \Delta-1}} \operatorname{Pr}\left[\vec{H}^{r \Delta-1}=\vec{h}^{r \Delta-1}\right] \cdot \frac{1}{\left|\mathcal{Y}_{\max }\right|} \cdot \operatorname{Adv}\left(y, \vec{h}^{r \Delta-1}\right) \\
= & \mathbb{E}\left[\operatorname{Adv}\left(U_{\mathcal{Y}_{\max }}, \vec{H}^{r \Delta-1}\right)\right] / 2,
\end{aligned}
$$

where the inequality is due to for any $y \in \mathcal{Y}_{\max }$ it holds that

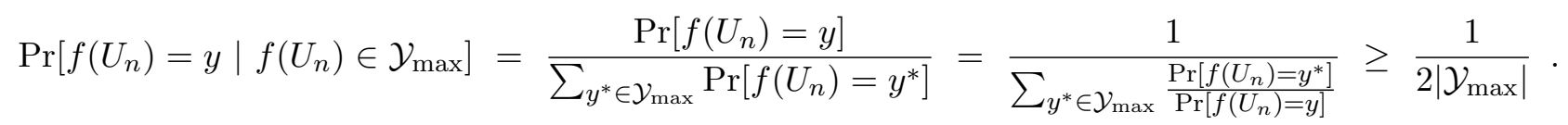

Proof of (18). For any $k \leq r \Delta$, we will define a $(n+1, r \Delta, \log |\mathcal{H}|)$-LBP $M_{1}$ that on input $x_{1}$ (at the source node) and $\vec{h}^{r \Delta-1}\left(h_{i} \in \mathcal{H}\right.$ at each $i^{t h}$ layer), outputs 1 iff every $t \in[k]$ satisfies $y_{t} \in \mathcal{Y}_{[\max ]}$. The $B S G 2^{-2 n}$-fools $M_{1}$, i.e., for any $x_{1} \in\{0,1\}^{n}$

$\left|\operatorname{Pr}\left[M_{1}\left(x_{1}, \vec{H}^{r \Delta-1}\right)=1\right]-\operatorname{Pr}\left[M_{1}\left(x_{1}, B S G\left(U_{q}\right)\right)=1\right]\right|=\left|\operatorname{Pr}\left[\mathcal{S}_{k} \mid X_{1}=x_{1}\right]-\operatorname{Pr}\left[\mathcal{S}_{k}^{\prime} \mid X_{1}=x_{1}\right]\right| \leq 2^{-2 n}$

and thus

$$
\operatorname{Pr}\left[\mathcal{S}_{k}^{\prime}\right] \geq \operatorname{Pr}\left[\mathcal{S}_{k}\right]-2^{-2 n} \geq 1-k \epsilon-2^{-2 n} .
$$

The bounded-space computation of $M_{1}$ is as follows: the source node input is $\left(y_{1} \in\{0,1\}^{n}, \operatorname{tag}_{1} \in\right.$ $\{0,1\}$ ), where $y_{1}=f\left(x_{1}\right)$ and $\operatorname{tag}_{1}=1$ iff $y_{1} \in \mathcal{Y}_{[\max ]}$ (or 0 otherwise). At each $i^{\text {th }}$ layer up to $i=k$, it computes $x_{i}:=h_{i-1}\left(y_{i-1}\right), y_{i}:=f\left(x_{i}\right)$ and sets $\operatorname{tag}_{i}:=1$ iff $\operatorname{tag}_{i-1}=1$ and $y_{i} \in \mathcal{Y}_{[\max ]}\left(\operatorname{tag}_{i}:=0\right.$ otherwise). Finally, $M_{1}$ produces $\operatorname{tag}_{k}$ as the final output.

Similarly, we define another $(n+1, r \Delta, \log |\mathcal{H}|)$-LBP $M_{2}$ that on input $\left(x_{1}, \vec{h}^{r \Delta-1}\right)$, outputs 1 iff $y_{k} \in$ $\mathcal{Y}_{\max }$, and thus

$$
\operatorname{Pr}\left[\mathcal{E}_{k}^{\prime}\right] \geq \operatorname{Pr}\left[\mathcal{E}_{k}\right]-2^{-2 n} \geq n^{-c}-2^{-2 n}
$$


The computation of $M_{2}$ is simply to compute $x_{i}:=h_{i-1}\left(y_{i-1}\right)$ and $y_{i}:=f\left(x_{i}\right)$ at each $i^{\text {th }}$ iteration and to output 1 iff $y_{k} \in \mathcal{Y}_{\max }$. It follows that

$$
\operatorname{Pr}\left[\mathcal{E}_{k}^{\prime} \wedge \mathcal{S}_{k}^{\prime}\right] \geq \operatorname{Pr}\left[\mathcal{E}_{k}^{\prime}\right]-\operatorname{Pr}\left[\neg \mathcal{S}_{k}^{\prime}\right] \geq n^{-c}-2^{-2 n}-\left(k \epsilon+2^{-2 n}\right) \geq n^{-c} / 2 .
$$

Proof of (19). For any $k \in[(r-1) \Delta]$, consider the following $(n+1, r \Delta, \log |\mathcal{H}|)$-LBP $M_{3}$ : on source node input $y_{1}=f\left(x_{1}\right)$ and layered input vector $\vec{h}^{r \Delta-1}$, it computes $x_{i}:=h_{i-1}\left(y_{i-1}\right), y_{i}:=f\left(x_{i}\right)$ at each $i^{\text {th }}$ layer. For iterations numbered by $(k+1) \leq i \leq(k+\Delta)$, it additionally sets $\operatorname{tag}_{i}=1$ iff either $\operatorname{tag}_{i-1}=1$ or $y_{i} \in \mathcal{Y}_{\max }$, where $\operatorname{tag}_{k}$ is initialized to 0 . Finally, $M_{3}$ outputs $\operatorname{tag}_{k+\Delta}$. By the bounded space generator we have

$$
\left|\operatorname{Pr}\left[M_{3}\left(X_{1}, \vec{H}^{r \Delta-1}\right)=1\right]-\operatorname{Pr}\left[M_{3}\left(X_{1}, B S G\left(U_{q}\right)\right)=1\right]\right|=\left|\operatorname{Pr}\left[\bigvee_{i=k+1}^{k+\Delta} \mathcal{E}_{i}\right]-\operatorname{Pr}\left[\bigvee_{i=k+1}^{k+\Delta} \mathcal{E}_{i}^{\prime}\right]\right| \leq 2^{-2 n},
$$

and thus by (13)

$$
\operatorname{Pr}\left[\bigvee_{i=k+1}^{k+\Delta} \mathcal{E}_{i}^{\prime}\right] \geq \operatorname{Pr}\left[\bigvee_{i=k+1}^{k+\Delta} \mathcal{E}_{i}\right]-2^{-2 n} \geq 1-n^{-\alpha}-2^{-2 n}
$$

Proof of (20). For any $k \in[r \Delta]$, consider the following $(2 n+1, r \Delta, \log |\mathcal{H}|)-\mathrm{LBP} M_{4}$ : on source node input $\left(y_{1}=f\left(x_{1}\right), y_{1}^{\prime}=f\left(x_{1}^{\prime}\right), \operatorname{tag}_{1} \in\{0,1\}\right)$, where $\operatorname{tag}_{1}=1$ iff both $y_{1}, y_{1}^{\prime} \in \mathcal{Y}_{[\max ]}$. For $1 \leq i \leq k$, at each $i^{\text {th }}$ layer $M_{4}$ computes $y_{i}:=f\left(h_{i-1}\left(y_{i-1}\right)\right), y_{i}^{\prime}:=f\left(h_{i-1}\left(y_{i-1}^{\prime}\right)\right)$ and sets $\operatorname{tag}_{i}=1$ iff $\operatorname{tag}_{i-1}=1 \wedge y_{i} \in \mathcal{Y}_{[\max ]} \wedge y_{i}^{\prime} \in \mathcal{Y}_{[\max ]}$. Finally, at the $(k+1)^{t h}$ layer $M_{4}$ outputs 1 iff $y_{k}=y_{k}^{\prime} \in \mathcal{Y}_{\max }$ (in respect for event $\mathcal{E}_{k} / \mathcal{E}_{k}^{\prime}$ ) and $\operatorname{tag}_{k}=1$ (in honor of $\mathcal{S}_{k} / \mathcal{S}_{k}^{\prime}$ ). Imagine running two iterates with random $x_{1}, x_{1}^{\prime}$ and seeded by a common hash function from distribution either $\vec{H}^{r \Delta-1}$ or $B S G\left(U_{q}\right)$, we have

$$
\begin{gathered}
\mathrm{CP}\left(Y_{k} \wedge \mathcal{E}_{k} \wedge \mathcal{S}_{k} \mid \vec{H}^{r \Delta-1}\right)=\underset{\left(x_{1}, x_{1}^{\prime}\right) \leftarrow U_{2 n}, \vec{h}^{r \Delta-1} \leftarrow \vec{H}^{r \Delta-1}}{\operatorname{Pr}}\left[M_{4}\left(x_{1}, x_{1}^{\prime}, \vec{h}^{r \Delta-1}\right)=1\right] \\
\mathrm{CP}\left(Y_{k}^{\prime} \wedge \mathcal{E}_{k}^{\prime} \wedge \mathcal{S}_{k}^{\prime} \mid B S G\left(U_{q}\right)\right)= \\
\underset{\left(x_{1}, x_{1}^{\prime}\right) \leftarrow U_{2 n}, \vec{h}^{r \Delta-1} \leftarrow B S G\left(U_{q}\right)}{\operatorname{Pr}}\left[M_{4}\left(x_{1}, x_{1}^{\prime}, \vec{h}^{r \Delta-1}\right)=1\right]
\end{gathered}
$$

and thus

$$
\begin{aligned}
& \left|\mathrm{CP}\left(Y_{k} \wedge \mathcal{E}_{k} \wedge \mathcal{S}_{k} \mid \vec{H}^{r \Delta-1}\right)-\mathrm{CP}\left(Y_{k}^{\prime} \wedge \mathcal{E}_{k}^{\prime} \wedge \mathcal{S}_{k}^{\prime} \mid B S G\left(U_{q}\right)\right)\right| \\
& \leq \mathbb{E}_{\left(x_{1}, x_{1}^{\prime}\right) \leftarrow U_{2 n}}\left[\left|\operatorname{Pr}\left[M_{4}\left(x_{1}, x_{1}^{\prime}, \vec{H}^{r \Delta-1}\right)=1\right]-\operatorname{Pr}\left[M_{4}\left(x_{1}, x_{1}^{\prime}, B S G\left(U_{q}\right)\right)=1\right]\right|\right] \\
& \leq 2^{-2 n} .
\end{aligned}
$$

It follows by (14) that

$$
\mathrm{CP}\left(Y_{k}^{\prime} \wedge \mathcal{E}_{k}^{\prime} \wedge \mathcal{S}_{k}^{\prime} \mid B S G\left(U_{q}\right)\right) \leq \mathrm{CP}\left(Y_{k} \wedge \mathcal{E}_{k} \wedge \mathcal{S}_{k} \mid \vec{H}^{r \Delta-1}\right)+2^{-2 n} \leq(r \Delta+1) \cdot 2^{\max -n+1} .
$$

Note that $y_{k}, \mathcal{E}_{k}^{\prime}$ and $\mathcal{S}_{k}^{\prime}$ depend only on $x_{1}$ and $\vec{h}^{r \Delta-1}$, namely, for any $\vec{h}^{k-1}$ and any $u_{1}, u_{2} \in B S G^{-1}\left(\vec{h}^{k-1}\right)$, $\mathrm{CP}\left(Y_{k}^{\prime} \wedge \mathcal{E}_{k}^{\prime} \wedge \mathcal{S}_{k}^{\prime} \mid U_{q}=u_{1}\right)=\mathrm{CP}\left(Y_{k}^{\prime} \wedge \mathcal{E}_{k}^{\prime} \wedge \mathcal{S}_{k}^{\prime} \mid U_{q}=u_{2}\right)=\operatorname{CP}\left(Y_{k}^{\prime} \wedge \mathcal{E}_{k}^{\prime} \wedge \mathcal{S}_{k}^{\prime} \mid B S G\left(U_{q}\right)=\vec{h}^{k-1}\right)$.

Therefore,

$$
\mathrm{CP}\left(Y_{k}^{\prime} \wedge \mathcal{E}_{k}^{\prime} \wedge \mathcal{S}_{k}^{\prime} \mid U_{q}\right)=\mathrm{CP}\left(Y_{k}^{\prime} \wedge \mathcal{E}_{k}^{\prime} \wedge \mathcal{S}_{k}^{\prime} \mid B S G\left(U_{q}\right)\right) \leq(r \Delta+1) \cdot 2^{\max -n+1} .
$$


Proof of (23). We have that

$$
\begin{aligned}
& \operatorname{CP}\left(\left(Y_{\left(j^{*}-1\right) \Delta+i^{*}}^{\prime}, U_{q}\right) \mid \mathcal{E}_{\left(j^{*}-1\right) \Delta+i^{*}}^{\prime} \wedge \mathcal{S}_{\left(j^{*}-1\right) \Delta+i^{*}}^{\prime}\right) \\
= & \frac{\operatorname{CP}\left(\left(Y_{\left(j^{*}-1\right) \Delta+i^{*}}^{\prime}, U_{q}\right) \wedge \mathcal{E}_{\left(j^{*}-1\right) \Delta+i^{*}}^{\prime} \wedge \mathcal{S}_{\left.\left(j^{*}-1\right) \Delta+i^{*}\right)}^{\prime}\right.}{\operatorname{Pr}\left[\mathcal{E}_{\left(j^{*}-1\right) \Delta+i^{*}}^{\prime} \wedge \mathcal{S}_{\left(j^{*}-1\right) \Delta+i^{*}}^{\prime}\right]^{2}} \\
\leq & \operatorname{CP}\left(Y_{\left(j^{*}-1\right) \Delta+i^{*}} \wedge \mathcal{E}_{\left(j^{*}-1\right) \Delta+i^{*}} \wedge \mathcal{S}_{\left(j^{*}-1\right) \Delta+i^{*}} \mid U_{q}\right) \frac{1}{\left(n^{-2 c} / 4\right) \cdot 2^{q}} \\
\leq & \frac{(r \Delta+1) \cdot 2^{\max -n+1}}{\left(n^{-2 c} / 4\right) \cdot 2^{q}} \leq \frac{16 r \Delta \cdot n^{2 c}}{2^{n-\max } \cdot 2^{q}}
\end{aligned}
$$

where the equality is similar to that in Fact A.1 (by renaming $\vec{H}^{r \Delta-1}$ to $U_{q}$ ), and the two inequalities are due to (18) and (20) respecitvely and thus

$$
\mathbf{H}_{2}\left(\left(Y_{(j-1) \Delta+i^{*}}^{\prime}, U_{q}\right) \mid \mathcal{E}_{(j-1) \Delta+i^{*}}^{\prime} \wedge \mathcal{S}_{(j-1) \Delta+i^{*}}^{\prime}\right) \geq n-\max +q-2 c \cdot \log n-\log r-\log \Delta-4 .
$$

The uniform distribution over $\mathcal{Y}_{\max } \times\{0,1\}^{q}$ has entropy

$$
\mathbf{H}_{2}\left(\left(U_{\mathcal{Y}_{\max }}, U_{q}\right)\right) \leq \log \left(\frac{1}{2^{-n+\max -1}} \cdot 2^{q}\right)=n-\max +q+1,
$$

and thus the entropy deficiency (i.e., the difference of two entropies above) $e \leq 2 c \log n+\log r+\log \Delta+5$.

Fact A.2 For any $\delta>-1$ and any positive integer $q$, it holds that

$$
(1+\delta)^{q} \geq 1+q \cdot \delta
$$

Proof. We prove by induction. For $q=1$ the equality holds. Suppose that the above holds for $q=k \in \mathbb{N}$, i.e., $(1+\delta)^{k} \geq 1+k \cdot \delta$, then for $q=k+1$ we have

$$
(1+\delta)^{k+1} \geq(1+k \cdot \delta)(1+\delta)=1+(k+1) \cdot \delta+k \delta^{2} \geq 1+(k+1) \cdot \delta
$$

which completes the proof.

\section{B Definitions, Explanations and Remarks}

Remark B.1 (some intuitions for $\mathcal{S}_{k}$ ) Throughout the proofs, we consider the (inverting, collision, etc.) probabilities conditioned on event $\mathcal{S}_{k}$, which requires that during the first $k$ iterations no $y_{i}(1 \leq i \leq$ $k$ ) hits the negligible fraction. This might look redundant as $\mathcal{S}_{k}$ occurs with overwhelming probability (by (12)). However, our proofs crucially rely on the fact that, as stated in (14), the collision probability of $y_{k}$ conditioned on $\mathcal{S}_{k}$ is almost the same (roughly $\tilde{O}\left(2^{\max -n}\right)$, omitting poly $(n)$ factors) as the ideal case, i.e., the collision probability of $f\left(U_{n}\right)$ conditioned on $f\left(U_{n}\right) \in \mathcal{Y}_{\max }$. This would not have been possible if not being conditioned on $\mathcal{S}_{k}$ even though $\mathcal{Y}_{\max +1}, \ldots, \mathcal{Y}_{n}$ only sum to a negligible function $\operatorname{negl}(n)$. To see this, consider the following simplified case for $k=1$, the collision probability of $y_{1}$ is equal to that of $f\left(U_{n}\right)$, and thus we have

$$
\frac{1}{2} \cdot \sum_{i=1}^{n} 2^{i-n} \cdot \operatorname{Pr}\left[f\left(U_{n}\right) \in \mathcal{Y}_{i}\right] \leq\left(\mathrm{CP}\left(f\left(U_{n}\right)\right)=\sum_{i=1}^{n} \sum_{y \in \mathcal{Y}_{i}} \operatorname{Pr}\left[f\left(U_{n}\right)=y\right]^{2}\right)<\sum_{i=1}^{n} 2^{i-n} \cdot \operatorname{Pr}\left[f\left(U_{n}\right) \in \mathcal{Y}_{i}\right]
$$

Suppose that there is some $\mathcal{Y}_{t}$ such that $t=\max +\Omega(n)$ and $\operatorname{Pr}\left[f\left(U_{n}\right) \in \mathcal{Y}_{t}\right]=\operatorname{neg}(n)$, then the above collision probability is of the order $O\left(2^{\max -n}\left(n^{-c}+2^{\Omega(n)} \operatorname{negl}(n)\right)\right.$. By setting negl $(n)=n^{-\log n}$, the collision probability blows up by a factor of $2^{\Omega(n)}$ than the desired case $\tilde{O}\left(2^{\max -n}\right)$, and thus unable to apply Lemma 3.1. In contrast, conditioned on $\mathcal{S}_{1}$ the collision probability is $\tilde{O}\left(2^{\max -n}\right)$. 
Definition B.1 (Collision probabilities conditioned on $\mathcal{S}_{k}^{\prime}$ and $\mathcal{E}_{k}^{\prime}$ ) In the derandomized version, we will use the following conditional collision probabilities, whose definitions (quite naturally extend the standard collision probabilities) are as follows:

$$
\begin{aligned}
\mathrm{CP}\left(Y_{k}^{\prime} \wedge \mathcal{E}_{k}^{\prime} \wedge \mathcal{S}_{k}^{\prime} \mid U_{q}\right) \stackrel{\text { def }}{=} \mathbb{E}_{u \leftarrow U_{q}}\left[\sum_{y} \operatorname{Pr}\left[f^{k}\left(X_{1}, \vec{H}^{r \Delta-1}\right)=y \wedge \mathcal{E}_{k}^{\prime} \wedge \mathcal{S}_{k}^{\prime} \mid{\overrightarrow{H^{\prime}}}^{r \Delta-1}=B S G(u)\right]^{2}\right] \\
\mathrm{CP}\left(Y_{k}^{\prime} \wedge \mathcal{E}_{k}^{\prime} \wedge \mathcal{S}_{k}^{\prime} \mid B S G\left(U_{q}\right)\right) \\
\stackrel{\text { def }}{=} \mathbb{E}_{{\overrightarrow{h^{\prime}}}^{r \Delta-1} \leftarrow B S G\left(U_{q}\right)}\left[\sum_{y} \operatorname{Pr}\left[f^{k}\left(X_{1}, \vec{H}^{\prime r \Delta-1}\right)=y \wedge \mathcal{E}_{k}^{\prime} \wedge \mathcal{S}_{k}^{\prime} \mid \vec{H}^{\prime r \Delta-1}={\overrightarrow{h^{\prime}}}^{r \Delta-1}\right]^{2}\right] \\
\quad \mathrm{CP}\left(Y_{k}^{\prime}, U_{q} \mid \mathcal{E}_{k}^{\prime} \wedge \mathcal{S}_{k}^{\prime}\right) \stackrel{\text { def }}{=} \sum_{(y, u)} \operatorname{Pr}\left[f^{k}\left(X_{1}, B S G\left(U_{q}\right)\right)=y \wedge U_{q}=u \mid \mathcal{E}_{k}^{\prime} \wedge \mathcal{S}_{k}^{\prime}\right]^{2} .
\end{aligned}
$$

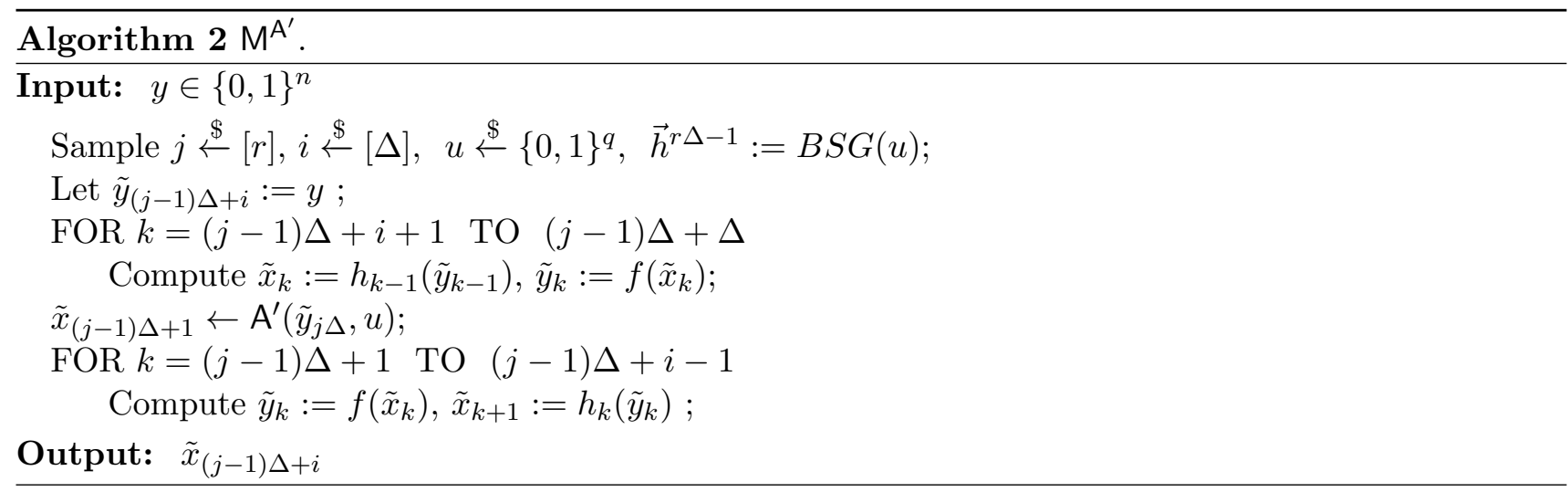

Remark B.2 (On weakening the condition of (1).) It is not hard to see from the proof that our construction only assumes a weaker condition than (1), i.e., for some constant $c \geq 0$ and $d=d(n) \in$ $O(\log n)$ it holds that

$$
\operatorname{Pr}\left[f\left(U_{n}\right) \in\left(\mathcal{Y}_{\max -d} \cup \mathcal{Y}_{\max -d+1} \cup \ldots \cup \mathcal{Y}_{\max }\right)\right] \geq n^{-c}
$$

We sketch the idea of adapting the proof to the relaxed assumption. By averaging there exists $t \in[0, d]$ such that $\mathcal{Y}_{\max -t}$ has weight at least $n^{-c-1}$. We thus consider the chance that $Y_{j}$ hits $\mathcal{Y}_{\max -t}$ (instead of $\mathcal{Y}_{\max }$ as we did in the original proof), and $O\left(n^{2 c+2} \cdot \omega(\log n)\right)$ iterations are bound to hit $\mathcal{Y}_{\max -t}$ at least once. Now we adapt the proof of Lemma 4.3. Ideally, conditioned on $f\left(U_{n}\right) \in \mathcal{Y}_{\max -t}$ the distribution $\left(f\left(U_{n}\right), \vec{H}^{r \Delta-1}\right)$ is uniform over $\mathcal{Y}_{\max -t} \times \mathcal{H}^{r \Delta-1}$ with full entropy

$$
\mathbf{H}_{2}\left(\left(U_{\mathcal{Y}_{\max -t}}, \vec{H}^{r \Delta-1}\right)\right) \leq \log \left(\frac{1}{2^{-n+\max -t-1}} \cdot|\mathcal{H}|^{r \Delta-1}\right)=n-\max +t+(r \Delta-1) \log |\mathcal{H}|+1 .
$$

However, we actually only have that

$$
\mathbf{H}_{2}\left(\left(Y_{(j-1) \Delta+i^{*}}, \vec{H}^{r \Delta-1}\right) \mid \mathcal{E}_{(j-1) \Delta+i^{*}} \wedge \mathcal{S}_{(j-1) \Delta+i^{*}}\right) \geq(n-\max +t+(r \Delta-1) \log |\mathcal{H}|+1)-e
$$

where entropy deficiency $e \leq t+O(\log n)=O(\log n)$. Then, we apply Lemma 3.1 and the hardto-invertness only blows up by a factor of roughly $2^{e}=n^{O(1)}$ than the ideal $\varepsilon$ (and taking a square root afterwards), which does not kill the iterate. Therefore, the iterate is hard to invert for every $O\left(n^{2 c+2} \cdot \omega(\log n)\right)$ iterations. The proof for the derandomized version can be adapted similarly. 


\section{Regular, Weakly-Regular and Arbitrary OWFs}

In this section, we discuss the gap between weakly-regular and arbitrary one-way functions. First, we show that most functions are known-almost-regular and thus weakly-almost-regular as well (see Remark 2.1), namely, "if a one-way function behaves like a random function, then it is known-almostregular". More generally, weakly-regular one-way functions cover a wider range of one-way functions (for positive $c \in \mathbb{N}$ ) than regular ones. We also (attempt to) characterize functions that are not captured by the definition of "weakly-regular". We show that in order not to fall into weakly-regular functions, the counterexamples should be somewhat artificial.

Now, we use probabilistic methods to argue that almost-regularity is a reasonable assumption in the average sense. That is, if the one-way function is considered as randomly drawn from the set of all (not just one-way) functions, then it is very likely to be almost-regular and thus a PRG can be efficiently constructed.

Lemma C.1 (A random function is known-almost-regular) Let $\mathcal{F}=\left\{f:\{0,1\}^{n} \rightarrow\{0,1\}^{m}\right\}$ be the set of all functions mapping $n$-bit to $m$-bit strings. For any $0<d<n$,

- if $m \leq n-d$, then it holds that

$$
\underset{f \stackrel{\Phi}{\leftarrow} \mathcal{F}}{\operatorname{Pr}}\left[\operatorname{SD}\left(f\left(U_{n}\right), U_{m}\right) \leq 2^{-d / 4}\right] \geq 1-2^{-d / 4}
$$

- if $m>n-d$, then we have

$$
\operatorname{Pr}_{\mathcal{F}, x \stackrel{\$}{\leftarrow}\{0,1\}^{n}}\left[1 \leq\left|f^{-1}(f(x))\right| \leq 2^{2 d+1}\right] \geq 1-2^{-d} .
$$

Typically, we can set $d \in \omega(\log n)$ so that $f$ will be almost regular except for a negligible fraction. Note that the first bullet gives even stronger guarantee than the second one does.

Proof of Lemma C.1. We see $\mathcal{F}$ as a family of universal hash functions and let $F$ be a uniform distribution over $\mathcal{F}$. For $m \leq n-d$ we have by the leftover hash lemma that

$$
\mathbb{E}_{f \leftarrow \mathcal{F}}\left[\mathrm{SD}\left(f\left(U_{n}\right), U_{m}\right)\right]=\mathrm{SD}\left(F\left(U_{n}\right), U_{m} \mid F\right) \leq 2^{-\frac{d}{2}} .
$$

It follows by a Markov inequality that the above statistical distance is bounded by $2^{-d / 2} \cdot 2^{d / 4}$ except for a $2^{-d / 4}$-fraction of $f$. We proceed to the case for $m>n-d$ to get

$$
\mathrm{CP}\left(F\left(U_{n}\right) \mid F\right) \leq \mathrm{CP}\left(U_{n}\right)+\max _{x_{1} \neq x_{2}}\left\{\operatorname{Pr}\left[F\left(x_{1}\right)=F\left(x_{2}\right)\right]\right\}=2^{-n}+2^{-m} \leq 2^{-n+d+1}
$$

We define $\mathcal{S} \stackrel{\text { def }}{=}\left\{(y, f):\left|f^{-1}(y)\right|>2^{2 d+1}\right\}$ to yield

$$
\begin{aligned}
2^{-n+d+1} & \geq \mathrm{CP}\left(F\left(U_{n}\right) \mid F\right)=\sum_{f} \operatorname{Pr}[F=f] \sum_{y} \operatorname{Pr}\left[f\left(U_{n}\right)=y\right]^{2} \\
\geq & 2^{-n+2 d+1} \cdot \sum_{f} \operatorname{Pr}[F=f] \sum_{y:(y, f) \in \mathcal{S}} \operatorname{Pr}\left[f\left(U_{n}\right)=y\right] \\
= & 2^{-n+2 d+1} \cdot \operatorname{Pr}\left[\left(F\left(U_{n}\right), F\right) \in \mathcal{S}\right],
\end{aligned}
$$

and thus $\operatorname{Pr}\left[\left(F\left(U_{n}\right), F\right) \in \mathcal{S}\right] \leq 2^{-d}$. This completes the proof. Note that $\left|f^{-1}(y)\right| \geq 1$ for any $y=f(x)$. 
BEYOND REGULAR FUNCTIONS. We cannot rule out the possibility that the one-way function in consideration is far from regular, namely (using the language of Definition 2.4), an arbitrary one-way function can have non-empty sets $\mathcal{Y}_{i}, \ldots, \mathcal{Y}_{i+O(n)}$. Below we argue that Definition 2.4 is quite generic and any function that fails to satisfy it should be somewhat artificial. As a first attempt, one may argue that if we skip all those $\mathcal{Y}_{j}^{\prime} s$ (in the descending order of $j$ ) that sum to negligible, the first one that is non-negligible ${ }^{9}$ (i.e., not meeting (2)) will satisfy (1) for at least infinitely many $n$ 's. In other words, an arbitrary one-way function is weakly-regular (at least for infinitely many $n$ 's). This argument is unfortunately problematic as (non-)negligible is a property of a sequence of probabilities, rather than a single value. However, we will follow this intuition and provide a remedied analysis below.

Lemma C.2 (a necessary condition to be a counterexample) Let $f:\{0,1\}^{n} \rightarrow\{0,1\}^{l(n)}$ be any one-way function and denote $\mathcal{Y}_{j} \stackrel{\text { def }}{=}\left\{y: 2^{j-1} \leq\left|f^{-1}(y)\right|<2^{j}\right\}$, and let $\kappa=\kappa(n)$ be the number of nonempty sets $\mathcal{Y}_{j}$ (that comprise the range of $f$ ) for any given $n$, and write them as $\mathcal{Y}_{i_{1}}, \mathcal{Y}_{i_{2}}, \ldots, \mathcal{Y}_{i_{\kappa}}$ with $i_{1}<i_{2}<\ldots<i_{\kappa}$. For every $n_{0} \in \mathbb{N} \cup\{0\}$, it must hold that function $\mu_{n_{0}}(\cdot)$ defined as

$$
\mu_{n_{0}}(n) \stackrel{\text { def }}{=}\left\{\begin{array}{cl}
\operatorname{Pr}\left[f\left(U_{n}\right) \in \mathcal{Y}_{i_{\kappa-n_{0}}}\right], & \text { if } \kappa>n_{0} \\
0, & \text { if } \kappa \leq n_{0}
\end{array}\right.
$$

is negligible. Otherwise (if the above condition is not met), there exists constant $c \geq 0, \max (n) \in \mathbb{N}$ and negligible function $\epsilon(n) \in[0,1]$ such that (2) holds (for all n's) and (1) holds for infinitely many $n$ 's.

Proof of Lemma C.2. If (26) does not hold for every $n_{0} \in \mathbb{N} \cup\{0\}$, then there must exist an $n_{0}$ such that $\mu_{0}(\cdot), \ldots \mu_{n_{0}-1}(\cdot)$ are negligible and $\mu_{n_{0}}(\cdot)$ is non-negligible. We then define $\max (\cdot)$ as

$$
\max (n) \stackrel{\text { def }}{=}\left\{\begin{array}{cl}
i_{\kappa(n)-n_{0},}, & \text { if } \kappa(n)>n_{0} \\
\perp, & \text { if } \kappa(n) \leq n_{0}
\end{array}\right.
$$

It is easy to see that $\mathcal{Y}_{i_{\kappa-n_{0}+1}}, \ldots, \mathcal{Y}_{i_{\kappa}}$ sum to a negligible fraction in $n$ (i.e., the sum of a finite number of negligible functions $\mu_{0}(\cdot), \ldots \mu_{n_{0}-1}(\cdot)$ results into another negligible function). Denote by $\mathcal{N}_{\perp} \stackrel{\text { def }}{=}\{n \in \mathbb{N} \cup\{0\}: \max (n)=\perp\}$. We have by assumption that for some constant $c$ it holds that $\mu_{n_{0}}(n) \geq n^{-c}$ for infinitely many $n \in \mathbb{N} \cup\{0\}$, and thus $\mu_{n_{0}}(n) \geq n^{-c}$ holds also for infinitely many $n \in \mathbb{N} \cup\{0\} \backslash \mathcal{N}_{\perp}$. This is due to $\mu_{n_{0}}(n)=0$ for any $n \in \mathcal{N}_{\perp}$. Therefore, $\operatorname{Pr}\left[f\left(U_{n}\right) \in \mathcal{Y}_{\text {max }}\right]$ is non-negligible, which completes the proof.

(26) IS A NECESSARY AND STRONG CONDITION. The above lemma formalizes a necessary condition to constitute a counterexample to Definition 2.4. It is necessary in the sense that any one-way function that does not satisfy it must satisfy Definition 2.4 (for at least infinitely many $n$ 's). Note that the condition is actually an infinite set of conditions by requiring every $\mu_{n_{0}}(n)$ (for $n_{0} \in \mathbb{N} \cup\{0\}$ ) being negligible. At the same time, it holds unconditionally that all these $\mu_{n_{0}}(n)$ (that correspond to the weights of all non-empty sets) must sum to unity, i.e., for every $n$ we have

$$
\mu_{0}(n)+\mu_{1}(n)+\ldots+\mu_{\kappa(n)-1}(n)=1 .
$$

The above might look mutually exclusive to (26) as if every $\mu_{n_{0}}(n)$ is negligible then the above sum should be upper bounded by $\kappa(n) \cdot \operatorname{negl}(n)=n e g l^{\prime}(n)$ instead of being unity. This intuition is not right in general, as by definition a negligible function only needs to be super-polynomially small for all sufficiently large (instead of all) $n$ 's. However, it is reasonable to believe that one-way functions satisfying (26) should be quite artificial.

\footnotetext{
${ }^{9}$ Although non-negligible and noticeable are not the same, they are quite close: a non-negligible (resp., noticeable) function $\mu(\cdot)$ satisfies that there exists constant $c$ such that $\mu(n) \geq n^{-c}$ for infinitely many (resp., all large enough) $n$ 's.
} 
(26) Is NOT SUfFICIEnT. Despite seeming strong, (26) is still not sufficient to make a counterexample. To show this, we give an example function that satisfies both (26) (for every $n_{0} \in \mathbb{N} \cup\{0\}$ ) and Definition 2.4. That is, let $f$ be a one-way function where for every $n$ the non-empty sets of $f$ are

$$
\mathcal{Y}_{n / 3}, \mathcal{Y}_{n / 3+1}, \ldots, \mathcal{Y}_{n / 2}
$$

with $\operatorname{Pr}\left[f\left(U_{n}\right) \in \mathcal{Y}_{n / 3}\right]=1-n^{-\log n+1} / 6, \operatorname{Pr}\left[f\left(U_{n}\right) \in \mathcal{Y}_{n / 3+i}\right]=n^{-\log n}$ for all $1 \leq i \leq n / 6$ and thus $\kappa(n)=n / 6+1$. It is easy to see that this function satisfies Definition 2.4 with $\max (n)=n / 3$ and $\epsilon(n)=n^{-\log n+1} / 6$. In addition, for every $n_{0} \in \mathbb{N} \cup\{0\}$ function $\mu_{n_{0}}(\cdot)$ is negligible as $\mu_{n_{0}}(n)=n^{-\log n}$ for all $n>6 n_{0}$. In summary, although an arbitrary one-way function may not be weakly-regular, the counterexamples must be well crafted to satisfy a somewhat artificial (yet still insufficient) condition. 